Petar Kassal' / Ema Horak ${ }^{1, a}$ / Marija Sigurnjak / Matthew D. Steinberg² / Ivana Murković Steinberg'

\title{
Wireless and mobile optical chemical sensors and biosensors
}

${ }^{1}$ Faculty of Chemical Engineering and Technology, University of Zagreb, Marulićev trg 19, HR-10000, Croatia, E-mail: ivana.murkovic@fkit.hr. https://orcid.org/0000-0003-1734-6107, https://orcid.org/0000-0001-7255-6612.

${ }^{2}$ CoSense Wireless Ltd, 57A Moorfield Road, Duxford, Cambridge CB22 4PP, UK

\begin{abstract}
:
This review explores the current state-of-the-art wireless and mobile optical chemical sensors and biosensors. The review is organised into three sections, each of which investigates a major class of wireless and/or mobile optical chemical sensor: (i) optical sensors integrated with a radio transmitter/transceiver, (ii) wearable optical sensors, and (iii) smartphone camera-based sensors. In each section, the specific challenges and tradeoffs surrounding the (bio)chemical sensing mechanism and material architecture, miniaturisation, integration, power requirements, readout, and sensitivity are explored with detailed examples of sensor systems from the literature. The analysis of 77 original research articles published between 2007 and 2017 reveals that healthcare and medicine, environmental monitoring, food quality, and sport and fitness are the target markets for wireless and mobile optical chemical sensor systems. In particular, the current trend for personal fitness tracking is driving research into novel colourimetric wearable sensors with smartphone readout. We conclude that despite the challenges, mobile and wearable optical chemical sensor systems are set to play a major role in the sensor Internet of Things.
\end{abstract}

Keywords: biosensors, chemical sensors, optical sensors, smartphone, wearable

DOI: 10.1515/revac-2017-0024

Received: November 16, 2017; Accepted: August 4, 2018

\section{Abbreviations}

CMOS complementary metal oxide semiconductor

DMSO dimethyl sulfoxide

FRET Förster resonance energy transfer

GSM Global System for Mobile Communications

IR infrared

ISM industrial, scientific and medical

LFIA lateral flow immunoassay

NFC near-field communication

PDMS Polydimethylsiloxane

pHEMA poly(2-hydroxyethyl methacrylate)

PMMA poly(methyl methacrylate)

RFID radiofrequency identification

RGB red, green, blue

SRD short-range devices

UV ultraviolet

Ivana Murković Steinberg is the corresponding author

a Present address: Computational Organic Chemistry and Biochemistry Group, Division of Organic Chemistry and Biochemistry, Ruđer Bošković Institute,

Bijenička cesta 54, HR-10000, Croatia

(C) 2018 Walter de Gruyter $\mathrm{GmbH}$, Berlin/Boston. 


\section{Introduction}

The demand for instant (bio)chemical information at the point of measurement combined with advances in mobile communication technologies is driving the development of wireless chemical sensors and biosensors for the sensor Internet of Things. These hybrid sensing devices collect analytical data on their local (bio)chemical environment, or perform spot tests on applied samples, and process and transmit this information to a remote device wirelessly, most usually by radio communications (Farre et al. 2009, Potyrailo et al. 2011, and Ghafar-Zadeh 2015). Common radio technologies used in such systems include Bluetooth, radiofrequency identification (RFID), ZigBee, near field communication (NFC), and 433- and 860-MHz short-range devices (ISM/SRD860) (Kassal, Steinberg \& Steinberg, 2018). Optical chemical sensors and biosensors lend themselves to integration into wireless systems thanks to their ease of miniaturisation, the unnecessary use of reference electrodes, the high detection sensitivity of certain modes (e.g., fluorescence), and the diversity of optically responsive indicator chemistries. In a radio-based wireless optical chemical sensor, the optical signal generated by the interaction of the analyte and receptor chemistry is converted to an electrical signal by the detector and is then passed to a remote wireless receiver or network where the data can be analysed in more detail (Figure $1 \mathrm{~A})$.

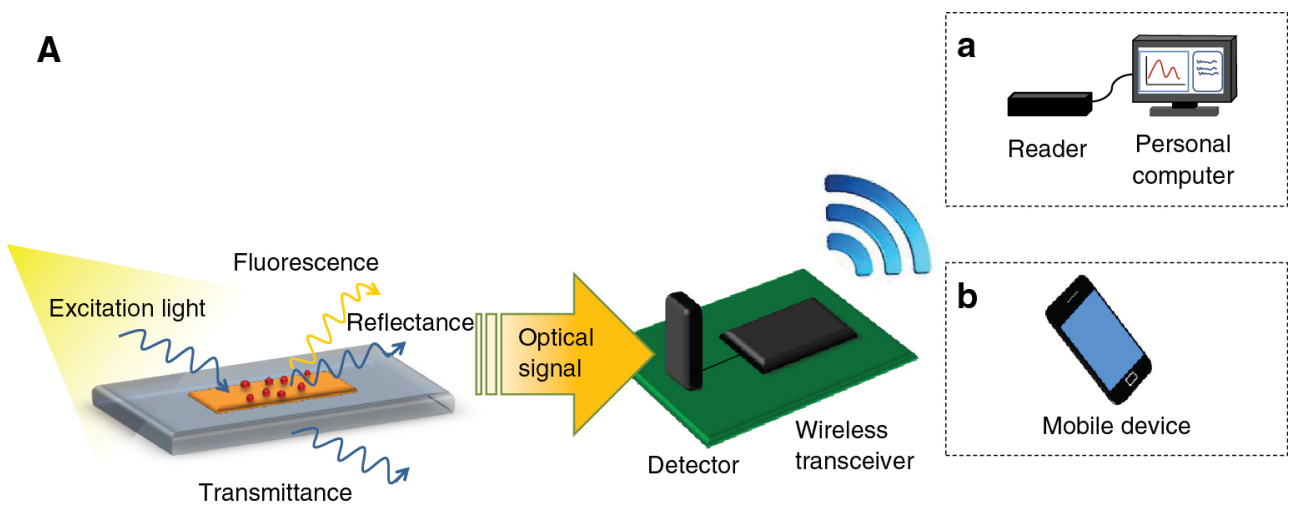

B
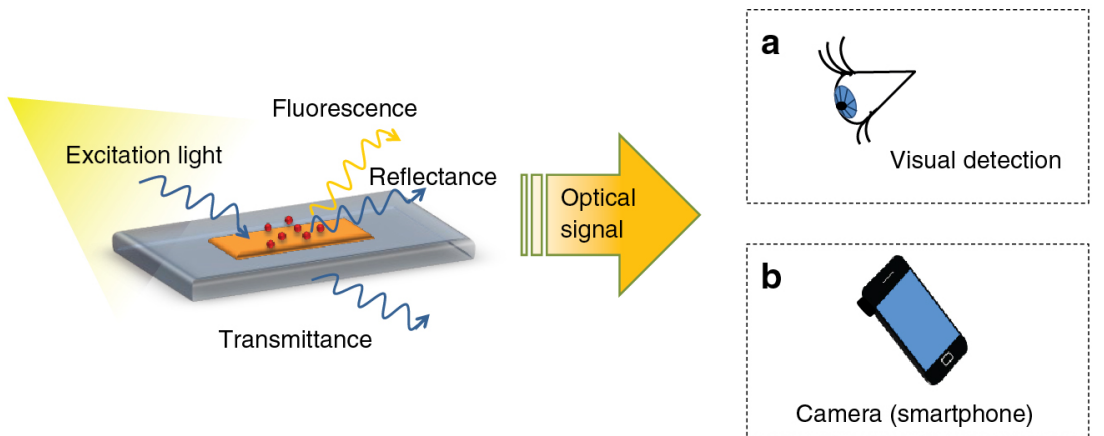

Figure 1: (A) Schematic showing the operation of a wireless optical (bio)chemical sensor. (B) Mobile optical sensors also enable wireless readout by the naked eye or a remote camera, without radio communication.

Wearable chemical sensing is becoming a key application area for wireless chemical sensors (Diamond et al. 2008) and has been the research focus of analytical chemists for many years. Several review papers have been published on electrochemical wearable sensors (Windmiller and Wang 2013, Bandodkar and Wang 2014, Steinberg, Kassal, and Steinberg 2016, and Malon Radha, Heng Lee, and Córcoles Emma 2017), but no similar review has yet been undertaken on optical wearable sensors. Wireless (radio-based) communication is often essential to ensure complete mobility of many types of wearable sensor, especially electrochemical devices (Steinberg, Kassal \& Steinberg, 2016). However, in a wearable optical system, the output signal can also be read by the naked eye or a camera (Figure 1B). Thus, both radio and visual readout systems are receiving attention from the academic community.

One mobile platform particularly being increasingly used in (bio)chemical sensing - the smartphone - has now become ubiquitous. According to the International Telecommunication Union (ITU), mobile cellular subscriptions reached 7.4 billion globally in 2016, almost reaching the number of people on Earth (ITU 2016). This pervasiveness, as well as the increasing computational ability of smartphones, makes them an ideal mobile platform for collection and analysis of (bio)chemical data (Zhang and Liu 2016). The smartphone can function 
as both a readout and a data analysis unit, typically using smartphone-enabled radio standards for readout such as Bluetooth, NFC, Wi-Fi, and Global System for Mobile Communications (GSM) (Figure 1A). Meanwhile, the increasing quality of integrated cameras enables the smartphone to also serve as the detector in optical (bio)chemical sensing systems (Figure 1B).

This review explores the current state-of-the-art and recent advances in wireless optical chemical sensors and biosensors, with emphasis on three major groups of mobile (bio)chemical systems: radio-based optical chemical sensors, optical chemical sensors that are wearable, and smartphone camera-based sensors. These groups are not mutually exclusive and the overlap is explained in Section 2.

\section{Methodology and structure}

Academic databases from the Web of Science were searched for chemical sensors and biosensors, using combinations of keywords indicating the sensors' wireless and mobile nature - "wireless," "contactless," "wearable," and "smartphone" - encompassing different modes of optical sensing, such as "absorbance" and "fluorescence." The initial literature search generated 281 publications (journal articles and conference proceedings) published from 2003 onwards (Figure 2). The scope of the review was then limited to research work in the area of wireless and mobile (bio)chemical sensing, which consequently reduced the number of publications to 77.

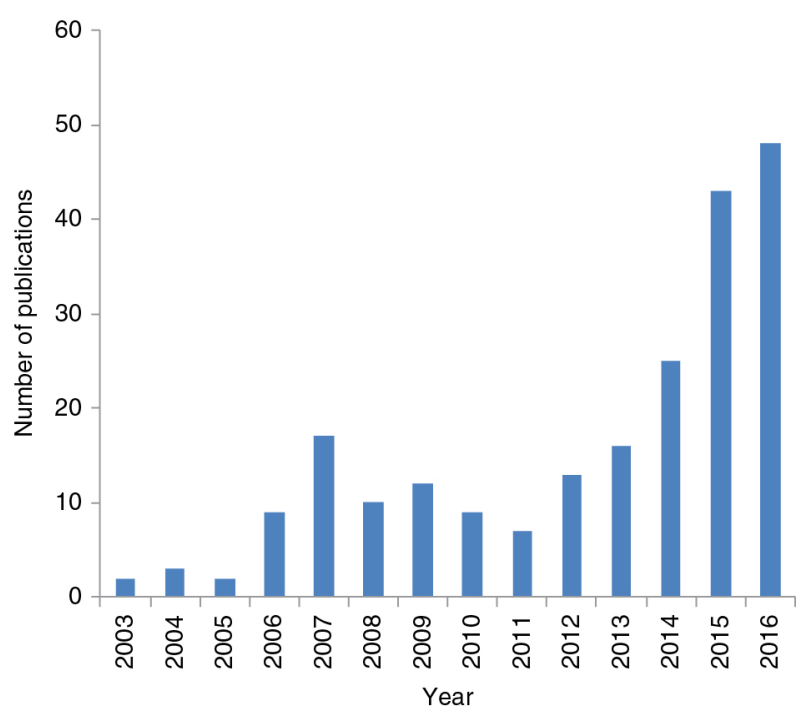

Figure 2: Number of publications per year for wireless, wearable, and smartphone-based optical chemical sensors and biosensors, retrieved from the Web of Science database.

To ensure the review is both topical and current, we define mobile sensing to include wearable and smartphone-based sensors - two extremely popular topics in analytical chemistry today. The growth of research in these fields is such that we present these in their own dedicated sections (Sections 4 and 5). However, mobile, handheld, and portable devices without wireless communication were deemed out of scope and are therefore not considered in our analysis. Further reading on these latter devices can be found elsewhere (Capitán-Vallvey and Palma 2011 and Choi 2016).

The review is thus structured in three parallel sections that may be read independently, each covering a major group of wireless and/or mobile chemical sensors. First, optical chemical sensors and biosensors integrated with a radio transmitter/transceiver are presented (Section 3). Second, optical wearable chemical sensors and biosensors are presented and discussed in Section 4. Although there is some overlap here with the former grouping, we think the recent explosion in academic research on wearable sensors and their applications justifies this subdivision. Clearly, the analytical data from a wearable optical sensor can be retrieved wirelessly by radio communication (Figure 1A) but may alternatively be retrieved by the naked eye or a remote camera (Figure 1B). Accordingly, we consider both wearable optical sensors with and without wireless (radio) data transfer. Lastly, chemical sensors and biosensors in which the optical signal is detected by smartphone camera are presented and discussed (Section 5). Although there are numerous examples of publications dealing with techniques for processing and analysing digital images in computer vision-based analytical procedures (Capitán-Vallvey et al. 2015), we have focused here on novel sensor chemistries and optical arrangements. Finally, the summary findings from all 77 research publications are presented (Section 6) together with our conclusions and future outlook on the field (Section 7). 


\section{Wireless optical sensors}

Optical chemical sensors are devices that respond to or generate optical signals when a chemical or biological analyte interacts with a receptor. In its simplest embodiment, an optical sensor might indicate the absorbance of an analyte in solution directly (or an analyte-sensitive indicator molecule), or for example, a more complex optical chemical sensor might indicate the luminescence intensity emitted by an indicator molecule in a receptor layer. In a radio-based wireless optical chemical sensor, the optical signal generated by the interaction of the analyte and receptor chemistry is converted to an electrical signal by the detector and is then passed to a remote wireless receiver or network where the data can be analysed in more detail (Kassal, Steinberg \& Steinberg, 2018).

In this section, we present a review of current academic research and development on optical chemical sensors and biosensors integrated with radio transceivers (Figure 1A), but which are not wearable. The wearable devices are presented separately in Section 4 in acknowledgement of the recent explosive growth in wearables in the past few years.

Wireless data transfer offers a significant advantage over wired systems, especially in applications where chemical analytes need to be monitored in places that are not easily accessible or hazardous. Therefore, wireless chemical sensors have found application mainly in environmental monitoring, for distributed air and water analysis, and in healthcare, as implantable or swallowable sensors. The sensors analysed in this section have been grouped by their application. Of the 77 publications covered by this review, 17 papers $(22 \%)$ were found to be based on wireless optical sensing, of which the main application areas were environmental monitoring $(47 \%)$ and healthcare $(24 \%)$.

\subsection{Environmental monitoring}

Wirelessly networked autonomous environmental analysers have been envisaged since the beginning of the millennium (Sequeira et al. 2002), and realisations of such systems quickly started to emerge. More than half of the wireless (bio)chemical sensors detected in the literature search have been developed for environmental monitoring applications, about evenly distributed between air quality and water quality monitoring (Table 1).

Table 1: Wireless optical chemical sensors for environmental monitoring.

\begin{tabular}{|c|c|c|c|c|c|c|}
\hline Analyte & Sample & $\begin{array}{l}\text { Detection } \\
\text { mode }\end{array}$ & $\begin{array}{l}\text { Detection } \\
\text { chemistry }\end{array}$ & $\begin{array}{l}\text { Immobilisation } \\
\text { method }\end{array}$ & $\begin{array}{l}\text { Wireless } \\
\text { communica- } \\
\text { tion }\end{array}$ & Reference \\
\hline $\begin{array}{l}\mathrm{pH} \text { (acetic acid } \\
\text { vapour) }\end{array}$ & Air & Absorbance & $\begin{array}{l}\text { Bromophenol } \\
\text { blue }\end{array}$ & $\begin{array}{l}\text { Ethyl } \\
\text { cellulose }\end{array}$ & ISM/SRD & $\begin{array}{l}\text { Shepherd et al. } \\
(2007)\end{array}$ \\
\hline $\mathrm{pH}\left(\mathrm{NH}_{3}\right.$ gas $)$ & Air & Absorbance & $\begin{array}{l}3^{\prime}-3^{\prime \prime}- \\
\text { Dichlorophenol } \\
\text { sulfonephthalein }\end{array}$ & Sol-gel & ZigBee & $\begin{array}{l}\text { Llorente-Alonso } \\
\text { et al. (2013) }\end{array}$ \\
\hline $\mathrm{NO}_{2}$ & Air & Absorbance & Diazo coupling & Porous glass & ISM/SRD & $\begin{array}{l}\text { Maruo et al. } \\
\text { (2012) }\end{array}$ \\
\hline Humidity & Air & $\begin{array}{l}\text { Absorbance } \\
\text { (IR) }\end{array}$ & Direct optical & - & ZigBee & Cao et al. (2014) \\
\hline Phosphate & Water & Absorbance & $\begin{array}{l}\text { Molybdovanado } \\
\text { phosphoric acid }\end{array}$ & $\begin{array}{l}\text { None - wet } \\
\text { chemistry }\end{array}$ & GSM & Slater et al. (2007) \\
\hline $\begin{array}{l}\mathrm{KMnO}_{4} \\
\text { (model) }\end{array}$ & Water & Absorbance & Direct optical & - & Bluetooth & $\begin{array}{l}\text { Camarillo- } \\
\text { Escobedo, } \\
\text { Valdés- } \\
\text { Perezgasga, and } \\
\text { Rodríguez-Rivera } \\
\text { (2013) }\end{array}$ \\
\hline $\mathrm{pH}$ & Water & Absorbance & $\begin{array}{l}\text { Bromocresol } \\
\text { purple }\end{array}$ & Ionogel film & Bluetooth & $\begin{array}{l}\text { Czugala et al. } \\
(2012)\end{array}$ \\
\hline $\mathrm{pH}$ & Water & Reflectance & $\begin{array}{l}\text { Universal } \\
\text { Indicator }\end{array}$ & $\begin{array}{l}\text { Paper, ethyl } \\
\text { cellulose }\end{array}$ & $\begin{array}{l}\text { Proprietary } \\
\text { (wireless } \\
\text { camera) }\end{array}$ & Fay et al. (2010) \\
\hline $\mathrm{NH}_{3}$ dissolved & Water & Fluorescence & $\begin{array}{l}\text { Eosin, } \\
\text { pH-sensitive } \\
\text { fluorophore }\end{array}$ & $\begin{array}{l}\text { Glass } \\
\text { substrate }\end{array}$ & Xbee & Deng et al. (2016) \\
\hline
\end{tabular}




\subsubsection{Air quality}

An early example of a wireless air quality sensor was an absorbance-based $\mathrm{pH}$ sensor for environmental acidity monitoring, which detected acetic acid plumes as model analyte (Shepherd et al. 2007). The system used a paired emitter - detector diode (PEDD) setup. In this setup, the light source is an LED and the detector is the same type of LED, but reverse biased. The photocurrent is measured indirectly by monitoring the discharge time of the detector in microseconds. Both LEDs were coated with ethyl cellulose doped with bromophenol blue (a $\mathrm{pH}$ indicator dye). The sensors were integrated with Crossbow wireless motes (ISM/SRD860), and several sensors were deployed to monitor plume movement. A more recent example for environmental acidity was developed by Llorente-Alonso et al. (2013) and tested with $\mathrm{NH}_{3}$ vapour. The sensor chemistry was developed by entrapping a $\mathrm{pH}$ indicator dye ( $3^{\prime}-3^{\prime \prime}$-dichlorophenol sulfonephthalein) within a sol-gel silicate film. The sensor uses an LED source and photodiode detector, and is accurate within $0.1 \mathrm{pH}$ units. It is integrated with a ZigBee module and should be applied to cultural heritage conservation.

Maruo et al. (2012) have developed a wide-area ubiquitous network based on Crossbow motes (IS$\mathrm{M} / \mathrm{SRD} 860$ ) in which bicycles equipped with mobile sensors collect $\mathrm{NO}_{2}$ concentration data in urban areas and transmit it wirelessly. The sensing element was porous glass doped with diazo coupling reagents, which selectively react with $\mathrm{NO}_{2}$ to produce an azo dye, the absorbance of which was measured with an LED and photodiode. The mobile sensors were equipped with a GPS unit that enabled the development of air quality maps, which could be sent directly to cyclists' mobile phones. The work of Cao et al. (2014) presents an example of a wireless chemical sensor that uses a laser source. The wireless humidity sensor represents a node in a ZigBee network and uses an IR laser diode and InGaAs detector to measure the infrared fingerprint of water. Apart from humidity, the system can be used for other gases. The sensor exhibits good linearity, stability, and selectivity, but the power consumption was not discussed.

\subsubsection{Water quality}

One of the earliest wireless water quality analysers was an autonomous field-deployable device for phosphate monitoring (Slater et al. 2007). Phosphate measurement was based on a microfluidic chip that mixes the reagent (molybdovanadophosphoric acid) and sample, after which the absorbance of the produced complex is measured with a LED and photodiode. The system was fairly large because it included reagent bottles, waste bottles, pumps, electronics, and a large battery; and power consumption was identified as a major issue. A GSM modem was used for wireless communication, exploiting the extensive coverage of the GSM network in Europe at the time. Collected data could also be sent in SMS messages to save power.

Camarillo-Escobedo, Valdés-Perezgasga, and Rodríguez-Rivera (2013) developed an autonomous optical microanalyser ( $\mu \mathrm{TAS}$ ) based on Bluetooth. Common pumps and injection valves were replaced by piezoelectric micropumps and microvalves, which bring the developed system closer to the autonomy and portability needed for real-time and in situ analysis. This work significantly reduced the reagent consumption, sample consumption, and waste generation, making a step towards green chemistry. The analyser can perform 2640 sampling cycles on a Li-ion battery. The system was tested with $\mathrm{KMnO}_{4}$ solutions, and a good comparison against a reference spectrophotometer was achieved. Czugala et al. (2012) developed a centrifugal portable system for in situ colourimetric analysis of water $\mathrm{pH}$ (Figure 3A). The system uses a PEDD setup, where an ionogel film, doped with bromocresol purple, is placed between the two diodes. Centrifugal disc technology removes the need for pumps, microfluidic interconnections, and fluid handling systems and can process multiple samples simultaneously. Bluetooth was used for wireless communication. A good comparison against a benchtop spectrophotometer was achieved. 
A
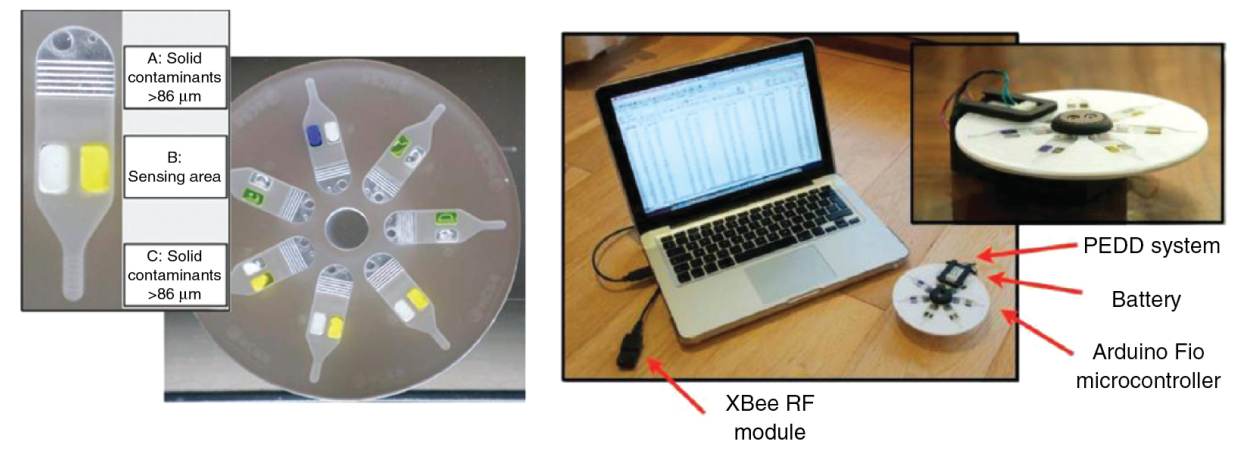

B

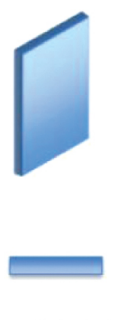

Substrate

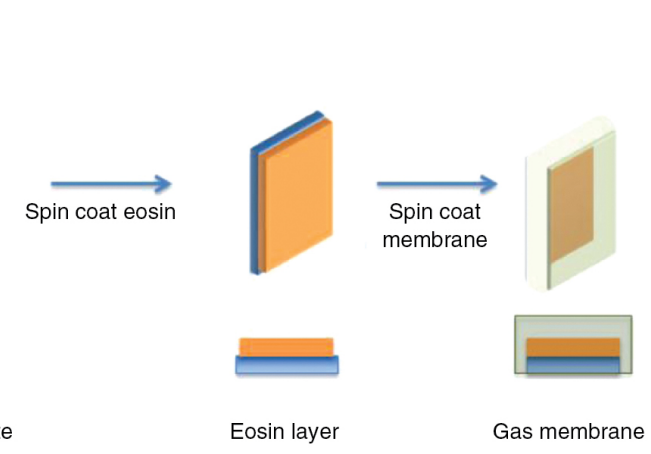

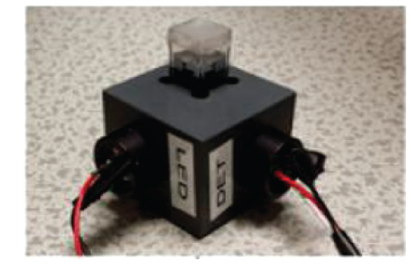

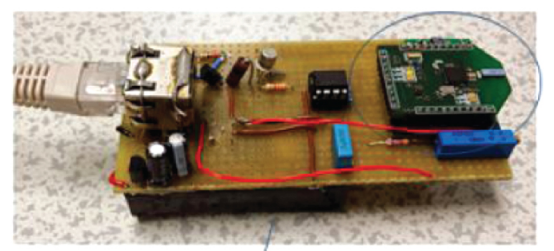

Figure 3: Wireless chemical sensors for environmental monitoring.

(A) Picture of the Lab-on-a-Disc device with a magnification of the fluidic channel with the sensing area, white (reference region) and yellow (ionogel sensor) (left); prototype of the wireless PEDD centrifugal microfluidic system (right). Adapted from Czugala et al. (2012). (B) Fluorescence-based ammonia sensor. Fabrication of the solid-state sensor (left); photographs of the optical sensor module and microcontroller-based electronics (right) (Deng et al. 2016).

A different approach to remote water quality monitoring has been demonstrated by Fay et al. (2010), who have developed a biomimetic robotic fish that can gather water $\mathrm{pH}$ data from multiple distributed sensing stations. The stations are equipped with a universal indicator strip, coated with ethyl cellulose to prevent leaching. The robotic fish has an on-board wireless camera that records colour of the stations and sends this information to a remote base station where local $\mathrm{pH}$ can be calculated with image processing software. Deng et al. (2016) have developed a fluorescence-based environmental sensor for dissolved ammonia (Figure 3B). The sensor uses eosin as the $\mathrm{pH}$-sensitive fluorescent dye that is immobilised on a glass substrate by a gas-permeable protection layer. Localised increases in ammonia concentration increase eosin fluorescence, which is detected with an LED and photodiode and transmitted to a base station using an Xbee module (proprietary radio module based on the IEEE 802.15.4 protocol).

\subsection{Healthcare}

The second largest application area of wireless chemical sensors is healthcare. The wireless optical sensors found in this group were highly miniaturised and mostly intended to be used as implantable and swallowable sensors. The main features of the sensors are summarised in Table 2, along with examples from other application areas (discussed in Section 3.3). 


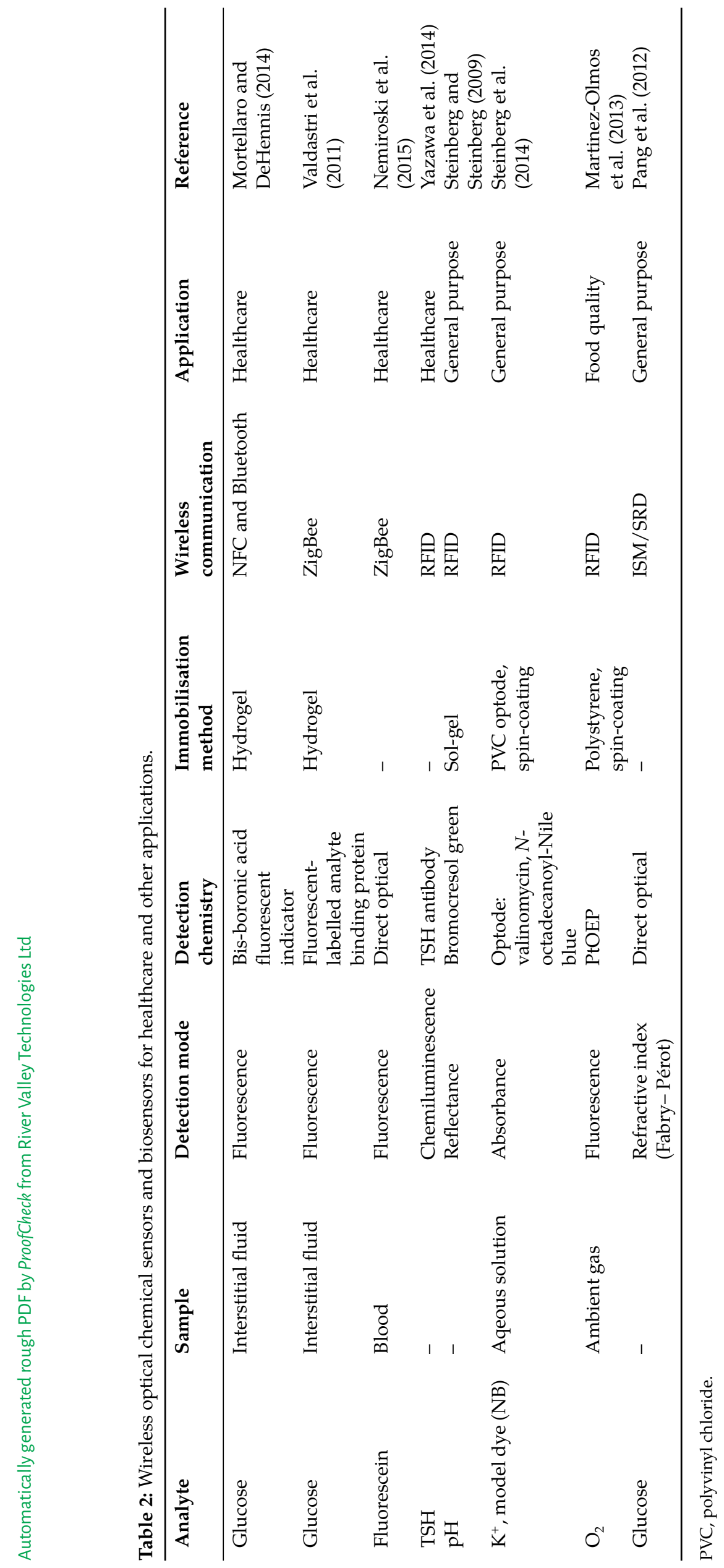


Mortellaro and DeHennis (2014) have described a subcutaneously implanted glucose sensor that uses a fluorescent, nonenzymatic glucose indicating hydrogel and a miniaturised optical detection system (Figure 4A). A fluorescent bis-boronic acid derivative is immobilised in the hydrogel (pHEMA). Upon binding of glucose, there is a disruption of the intramolecular photo-induced electron transfer, which causes increased fluorescence intensity. This change in fluorescence is detected with a UV LED and two photodiodes. The sensor is passiveit is powered by and communicates with a body-worn reader via NFC. The reader is equipped with a Bluetooth module that transmits over longer distances, and it has a battery that can simply be recharged at will. The sensor is not subject to the stability limitations inherent to enzyme-based systems and is less affected by biofouling than electrochemical sensors because the signal is derived from the entire bulk of the hydrogel and not just the surface. The wireless sensor was implanted and tested in vivo during a period of 28 days. Another example of an implantable fluorescent-based glucose sensor has been demonstrated in the literature (Valdastri et al. 2011). It contains a hydrogel with immobilised analyte binding protein covalently linked to two fluorescent proteins. Analyte-induced changes in FRET between two fluorescent proteins are detected with a laser diode source and phototransistor detectors. Because of the large power consumption of the laser diode, half of the volume of this implantable sensor is occupied by a battery, which hinders possibility of implantation.

A
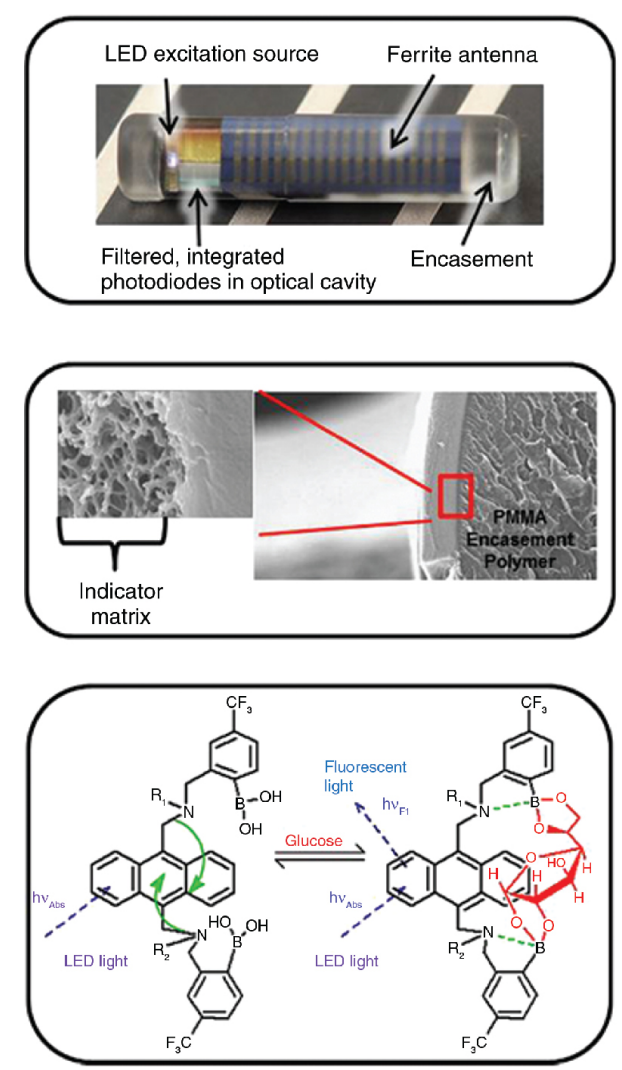

B

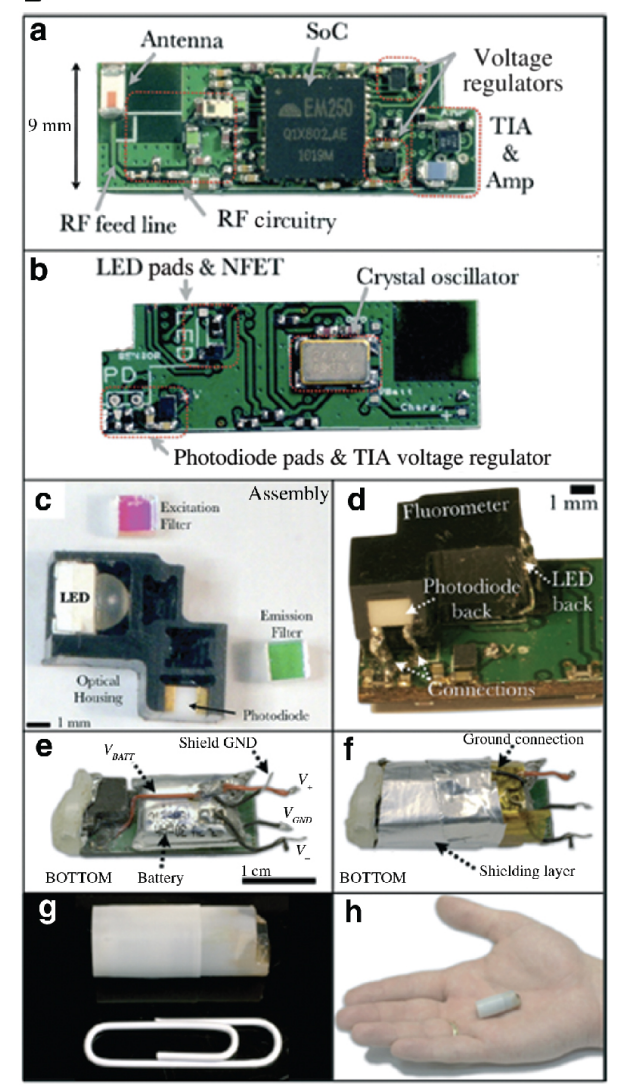

Figure 4: Wireless chemical sensors for healthcare applications.

(A) Photograph of the implantable glucose sensor (top); scanning electron microscope (SEM) images of the glucose indicator hydrogel grafted onto the outside of the PMMA sensor encasement (middle); and chemical structure and glucose binding mode of indicator moiety (bottom). R2 shown in the figure denotes connectivity to the hydrogel backbone, whereas R1 represents a propionic acid side chain. Adapted from Mortellaro and DeHennis (2014). (B) Photographs of the capsular fluorometer. (A-B) Top and bottom of the assembled four-layer PCB that houses all electronic components. (C) Optical components and optical housing of the fluorometer. (D) Mounting of assembled optical sensor onto the PCB. (E) Complete device without encapsulation. (F) Device with electromagnetic shielding. Encapsulated fluorometer pictured next to a standard paperclip $(\mathrm{G})$ and in the palm of a hand $(\mathrm{H})$ (Nemiroski et al. 2015).

A swallowable wireless capsule containing a miniature fluorimeter has been developed to monitor gastrointestinal bleeding (Figure 4B) (Nemiroski et al. 2015). The fluorimeter detects the presence of a leaked fluorescent dye (fluorescein), previously injected into the blood stream, in the gastrointestinal tract. Bulky focusing optics were eliminated by using pinholes to form a $0.2-\mu \mathrm{L}$ detection volume in which $20 \mathrm{nM}$ of fluorescein could be detected. The capsule transmits data to an external unit via the ZigBee protocol and can operate for up to several days, depending on the measurement and transmission rates. Lastly, an example of a wireless biosensor using chemiluminescence was demonstrated (Yazawa et al. 2014). The system is based on a passive RFID tag with an 
integrated photodiode that measures luminescence in a microfluidic channel. The sensor was used to detect thyroid-stimulating hormone (TSH) in a sandwich microfluidic immunoassay. The sensor itself consumes very little power, but the microfluidics are operated separately; and the measurement procedure is very long and uses several reagent additions and washing steps.

\subsection{Other applications}

A highly integrated passive RFID platform using dual-wavelength optical absorption for chemical sensing was introduced by Steinberg and Steinberg (2009) (Figure 5A). The system uses two LEDs and a photodiode in a planar configuration. The sensing chemistry used in the proof-of-concept demonstration was a sol-gel silicate thin film with an immobilised $\mathrm{pH}$-sensitive dye bromocresol green. A good correlation of the $\mathrm{pH}$ response against a laboratory spectrophotometer was demonstrated in the 5.2-8.3 range. The performance of the optical RFID tag was tested further in absorptiometric (transmission) mode, in two model analytical applications: photometry in solution, where dye concentration was determined, and potassium sensing with valinomycin-based bulk optode membranes (Steinberg et al. 2014). In both cases, the results correlated well with those obtained with a reference spectrophotometer.

A

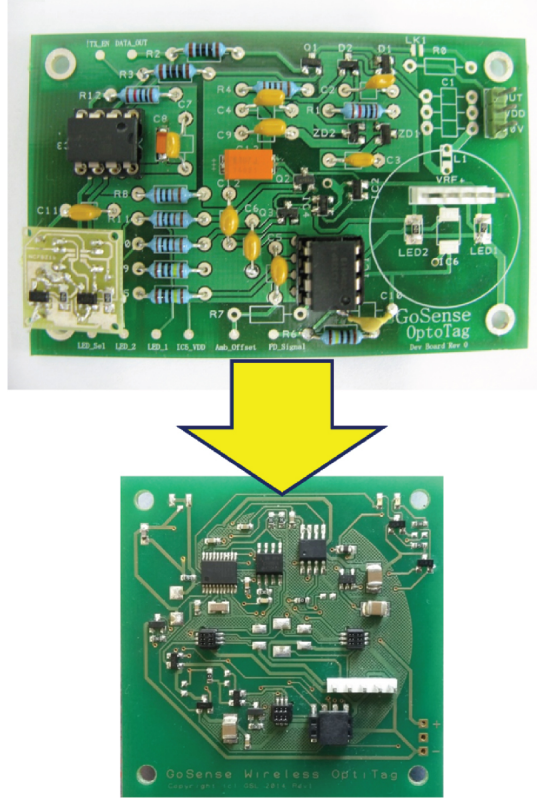

B

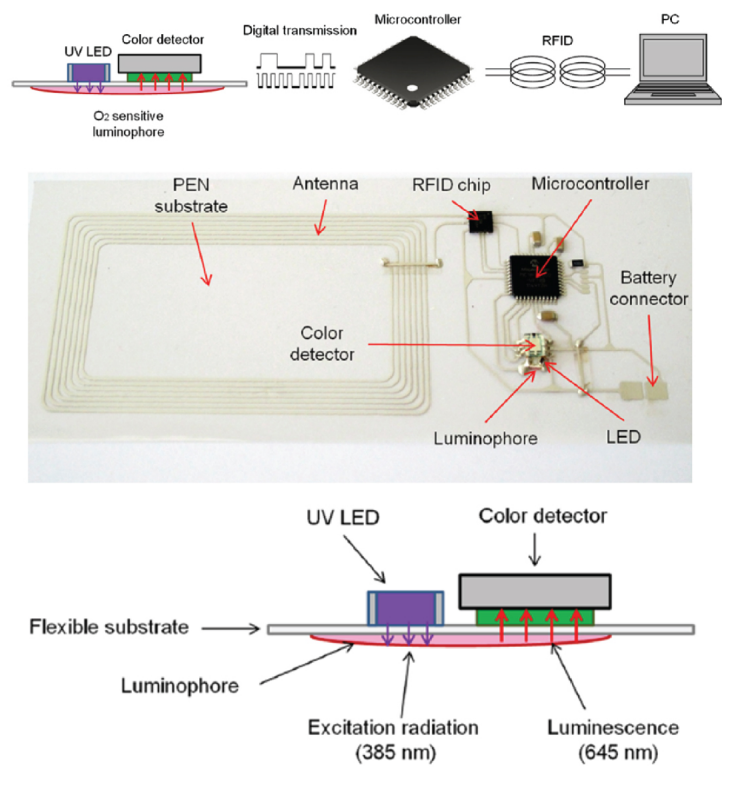

Figure 5: (A) Evolution of the wireless RFID platform for optical chemical sensing: the original (top), adapted from Steinberg et al. (2014); as used in Kassal et al. (2017) (bottom). (B) Operation of the wireless system for oxygen monitoring in packaged food (top); photograph of the system (middle); side view of the sensing module (bottom). Adapted from Martinez-Olmos et al. (2013).

Martinez-Olmos et al. (2013) developed a passive RFID-based fluorescent $\mathrm{O}_{2}$ sensor for monitoring the freshness of packaged food (Figure 5B). The RFID antenna was created by screen printing on one side of a polyester substrate; and the other electronic components, including an UV LED source and RGB detector, were added to the same side. A polystyrene film containing platinum octaethylporphyrin (PtOEP) was placed on the other side. Fluorescence of PtOEP is quenched by oxygen that can be detected through the polyester packaging. The system exhibited a resolution of $0.1 \mathrm{ppm}$, an accuracy better than $0.1 \%$, and an LOD of $40 \mathrm{ppm}$.

Pang et al. (2012) have developed an optical wireless sensor network, based on fiberoptic Fabry-Pérot sensors and Crossbow motes. The multisensor platform consisted primarily of optical physical sensors (temperature and pressure sensors), but chemical sensing (through refractive index changes of the sample solution) was also demonstrated with glucose as the model analyte. 


\section{Wearable optical sensors}

Wearable chemical sensors represent a specific subgroup of wireless sensors. Their application requires miniaturisation and integration into wearable accessories, into clothing, and into body art as wearables for personal biomonitoring and personal microenvironment monitoring (Steinberg, Kassal \& Steinberg, 2016).

As mentioned in the Introduction, wearable sensors have early been identified as a major application area of wireless chemical sensors (Diamond et al. 2008). In recent years, wearable (bio)chemical sensors have become very popular because they enable real-time and noninvasive monitoring of a multitude of healthcareand fitness-associated analytes (Bandodkar and Wang 2014 and Matzeu, Florea, and Diamond 2015). Although a significant amount of wearable electrochemical sensors is present in the literature, optical detection in wearable systems is less popular. The main reason for this might be the need for the light source and detector, which are rigid components and are challenging to implement into a wearable system (Windmiller and Wang 2013). Nevertheless, optical wearable sensors are present in the literature and are discussed here. Of the 77 publications covered by this review, 17 papers (22\%) could be classified as wearable optical sensors. Of the wearable sensors, $29 \%$ had integrated electronics for radio communication. The main application areas of wearable sensors were found to be sport and fitness $(41 \%)$ and healthcare $(35 \%)$.

\subsection{Wearable optical sensors for sport and fitness applications}

A particularly promising area for wearable chemical sensing is real-time perspiration analysis (Steinberg, Kassal \& Steinberg, 2016). Sweat is easily accessible and contains multiple analytes that are important for sport physiology (Bandodkar and Wang 2014). Therefore, all of the wearable sensors discussed in this section use sweat as the sample. Other features of the wearable sensors for sweat sensing are summarised in Table 3.

Caldara et al. (2012) have developed a textile-based wearable optical $\mathrm{pH}$ sensor. The sensor consists of cotton fabric with a $\mathrm{pH}$ indicator methyl red immobilised in a hybrid sol-gel (ormosil) matrix. The miniaturised wearable electronics included colour detection optoelectronics (white LED and photodiode) and microcontroller. The sensor exhibits low power consumption and resolution of $0.05 \mathrm{pH}$ units with dynamic range from $\mathrm{pH} 4$ to 8 , which is suitable for sweat $\mathrm{pH}$ analysis. The system was improved by covalent immobilisation of litmus as a $\mathrm{pH}$ indicator into a hybrid sol-gel matrix and using Bluetooth for wireless data transfer (Caldara et al. 2016). The authors emphasise much shorter times for colour transition in comparison to methyl red dye used in previous work. On-body $\mathrm{pH}$ measurements during physical activity were carried out. The operational lifetime was determined to be $24 \mathrm{~h}$ while sampling every $5 \mathrm{~s}$ and transmitting data every $2 \mathrm{~min}$, which is adequate for physical activity monitoring. A textile-based sensor incorporating a microfluidic platform with passive pumping and channels for collecting sweat was developed by Morris et al. (2009). The sweat $\mathrm{pH}$ measurements were carried out by paired emitter-detector LEDs, where the LEDs were at an angle and measured reflectance from a $\mathrm{pH}$-sensitive layer with immobilised bromocresol purple. On-body trials of sweat $\mathrm{pH}$ during physical exercise were performed using a waistband and a Crossbow mote enabled wireless communication. The system was optimised by substituting the LED detector with a photodiode and operating in transmission mode to increase sensitivity, improving the microfluidics by introducing a cotton thread to ensure fast and homogeneous flow, and by using Bluetooth communication (Curto et al. 2012) (Figure 6A). 


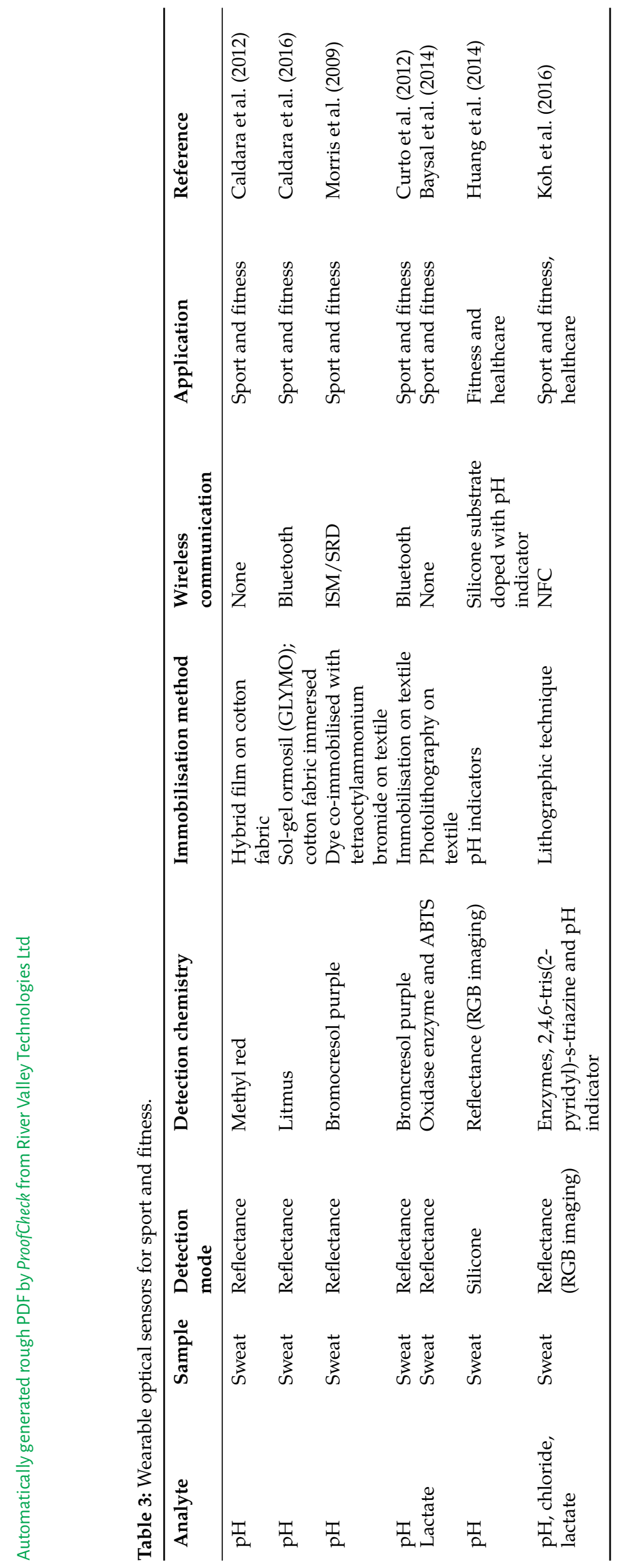


A

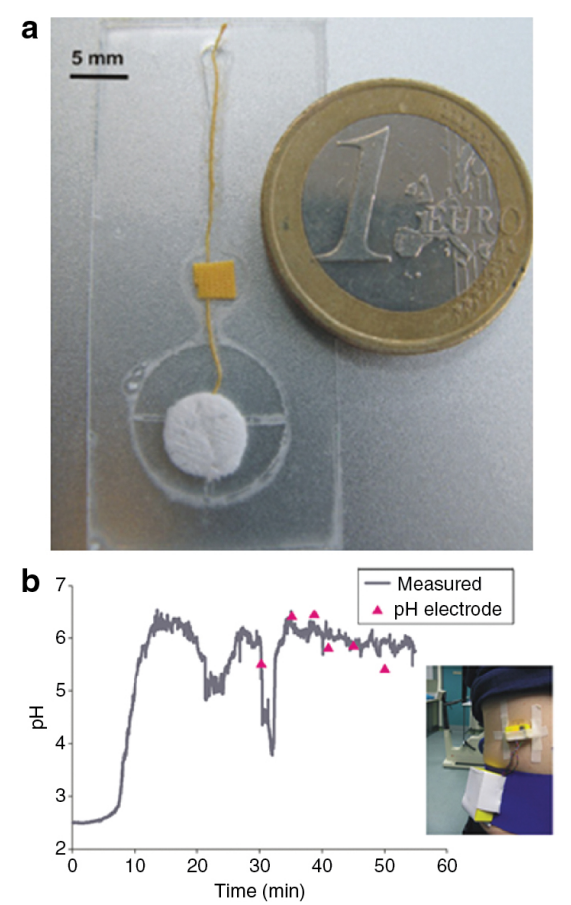

\section{B}

a

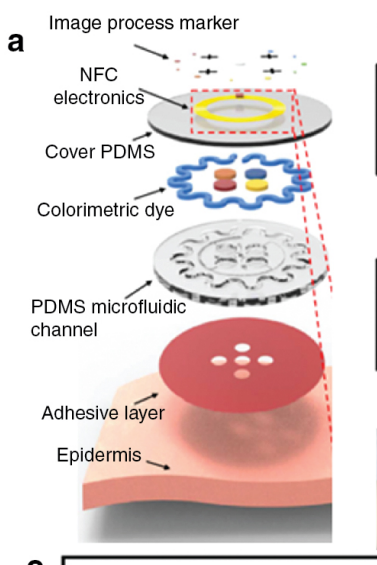

b
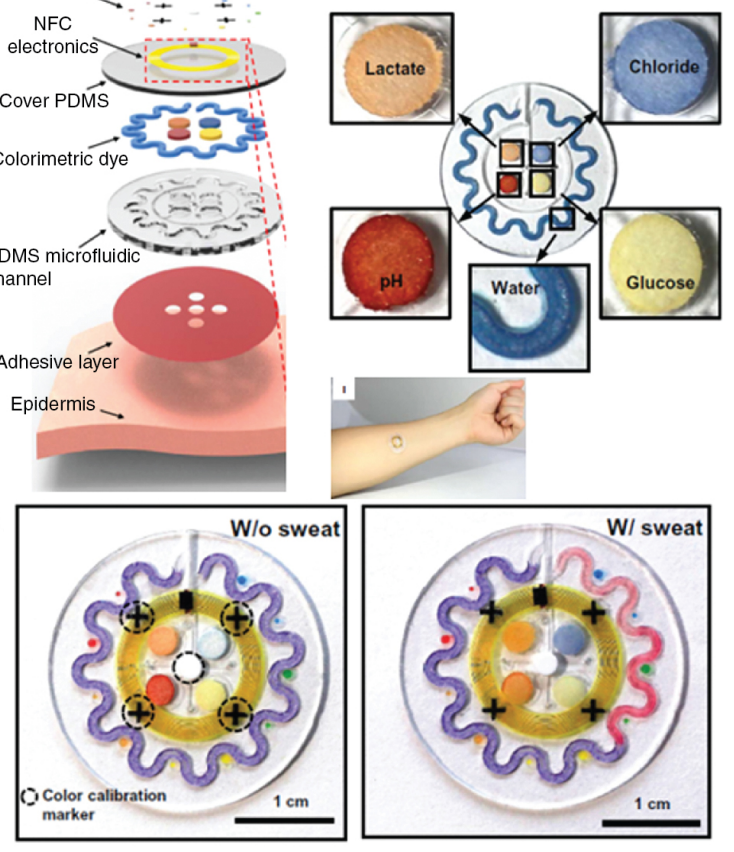

Figure 6: (A) Microfluidic chip for sweat collection (a) and on-body pH measurement (b) (Curto et al. 2012). (B) Schematic illustration of an epidermal microfluidic sweat monitoring device (a); colourimetric detection reservoirs that enable determination (b); images of the epidermal microfluidic biosensor (left) before and (right) after injecting artificial sweat (c), (Koh et al. 2016).

Baysal et al. (2014) presented a textile-based sensor for lactate analysis in sweat. An absorbent biocompatible nonwoven substrate was used for fabrication and design of microchannels and reservoirs as well as hydrophobic barriers by photolithography. The obtained porous structure resulted in capillary flow of sweat. Lactate concentration was determined by an oxidase enzyme-specific reaction. Two enzymes were added to the reservoir, namely, lactate oxidase and peroxidase and 2,2'-azino-bis (3-ethylbenzothiazoline-6-sulfonic acid) diammonium salt (ABTS). Oxidation of lactic acid produces hydrogen peroxide that leads to oxidation of ABTS, resulting in change of colour from green to brown. Huang et al. (2014) designed stretchable and wireless electrical (dielectric) sensors for volumetric sweat measurement with the possibility of both colourimetric and electrical analysis. Silicone substrates with immobilised $\mathrm{pH}$ indicator dyes show visible colour change in buffer solutions of different $\mathrm{pH}$. The authors also demonstrated the detection of iron and copper ions with commercial colourimetric kits. Koh et al. (2016) went a step further and developed a soft, flexible, and stretchable microfluidic system for simultaneous detection of four analytes in sweat: $\mathrm{pH}$, chloride, lactate, and glucose, as well as sweat volume and sweat rate (Figure 6B). This microfluidic platform is composed of three parts: (i) an adhesive layer with openings for sweat capture, (ii) a complement of channels and reservoirs with reagents for paper-based colourimetric analysis, and (iii) electronics that include a magnetic loop antenna for wireless NFC. Sweat is captured in a set of microchannels and reservoirs that use capillary action and natural pressure to move towards separate regions for analysis. The smartphone camera is initialised automatically when near the device using NFC and captures a digital image. On-body human trials proved high biocompatibility with no discomfort or skin irritation.

\subsection{Wearable optical sensors for healthcare and other applications}

\subsubsection{Wound monitoring}

Wound monitoring has been identified as a key application area of wearable sensors in healthcare (Steinberg, Kassal \& Steinberg, 2016). In wound monitoring scenarios, wireless data transfer should enable truly noninvasive wound assessment, which is of paramount importance in reducing patient discomfort (Mehmood et al. 2014). $\mathrm{pH}$ of wound fluid is an important wound status biomarker, and all of the optical wound sensors found in the literature detect wound $\mathrm{pH}$ as the analyte (Table 4).

Pasche et al. (2009) have developed a sensor with pH-responsive hydrogels and covalently immobilised phosphorylcholine receptors incorporated into optical fibers for detection of $\mathrm{pH}$ and C-reactive protein con- 
centration in wound. Surface functionalisation of the fiber was obtained using a photoactive dextran-based coating $\left(O p t o D e x^{\circledR}\right)$ [Arrayon Biotechnology (Neuchâtel, Switzerland)] covalently bound on to the surface, enabling specific covalent attachment of the target biomolecule. The portable sensing system contains optical fibers connected to the light source (LED) and the detector, which allows monitoring of the change in the refractive index at the surface of the fiber. Poly(methyl methacrylate) (PMMA) fibers show great potential for use in textile-based sensing systems as they can be woven into textiles. Schyrr et al. (2014) have developed a mobile optical fiber sensor for $\mathrm{pH}$ wound measurement. They obtained the sensor by coating PMMA optical fibers with a sol-gel film containing a $\mathrm{pH}$-sensitive dye. $\mathrm{pH}$ detection was provided with portable sensing device with light coupler and LED measuring light absorption variation in the coating. The sensor was tested in blood serum samples and manifested reversibility, a precision of $0.2 \mathrm{pH}$ units, and a response time of $20 \mathrm{~min}$.

We have reported on a wireless smart bandage for optical wound $\mathrm{pH}$ detection (Figure 7A) (Kassal et al. 2017). A pH indicator dye was covalently bound to cellulose particles dispersed within a biocompatible hydrogel, which enables production of $\mathrm{pH}$-sensitive layers on different kinds of dressing materials. Detection was provided with an optoelectronic probe and a miniature wireless platform that measures and stores $\mathrm{pH}$ data and transfers them to a remote computer using RFID. The results show high accuracy and precision of the smart bandage in the relevant $\mathrm{pH}$ range and could provide insight into temporal $\mathrm{pH}$ changes of wounds during the healing process. Tamayol et al. (2016) have developed hydrogel fibers for epidermal $\mathrm{pH}$ detection. $\mathrm{pH}$-responsive microbeads, loaded with brilliant yellow, were dispersed within the alginate-based microfibers using a microfluidic spinning method that enabled manipulation of the fiber size. The produced fibers were used as a medical tape-based wound dressing and the $\mathrm{pH}$ was measured via smartphone image capture. 


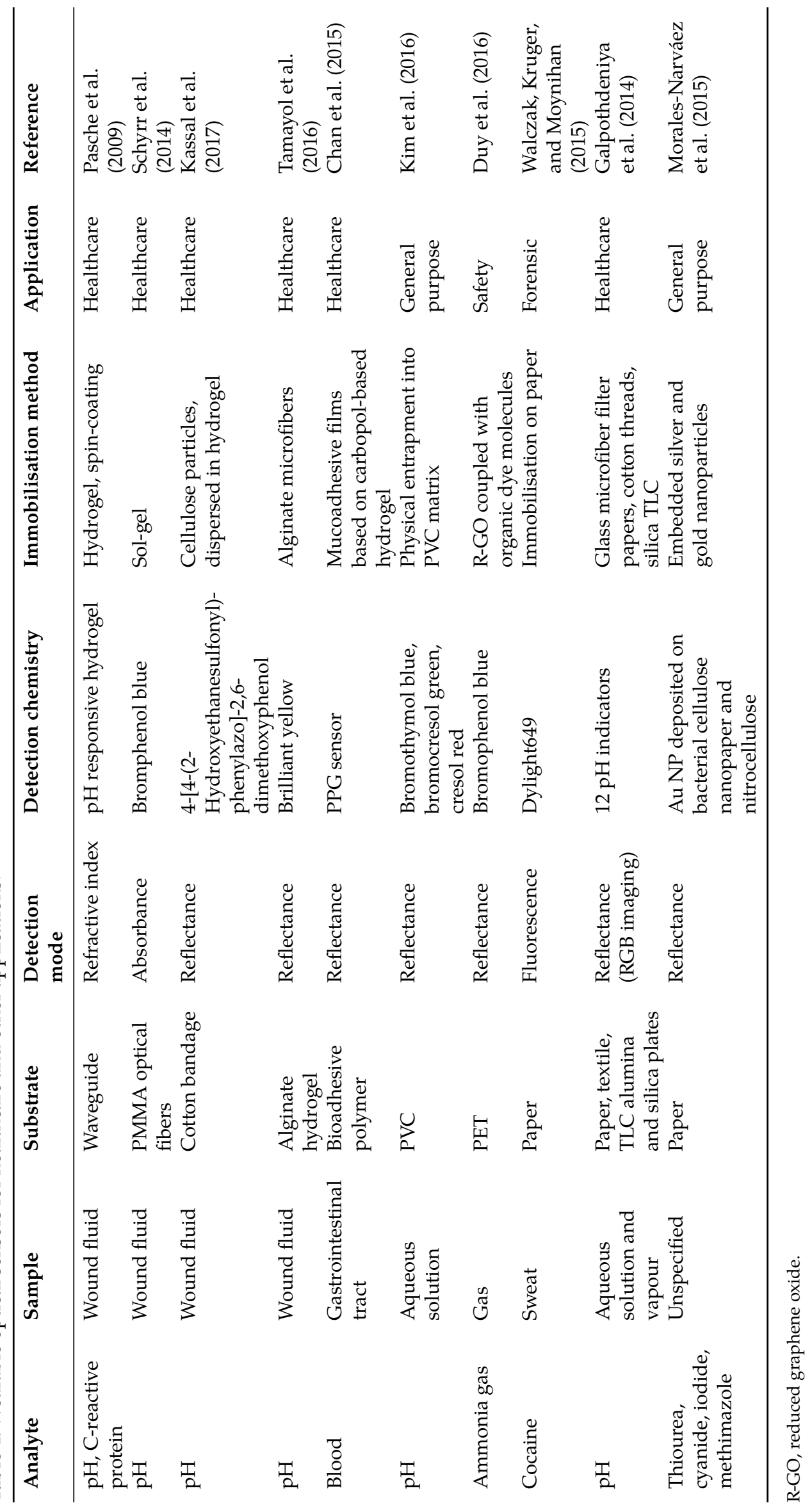




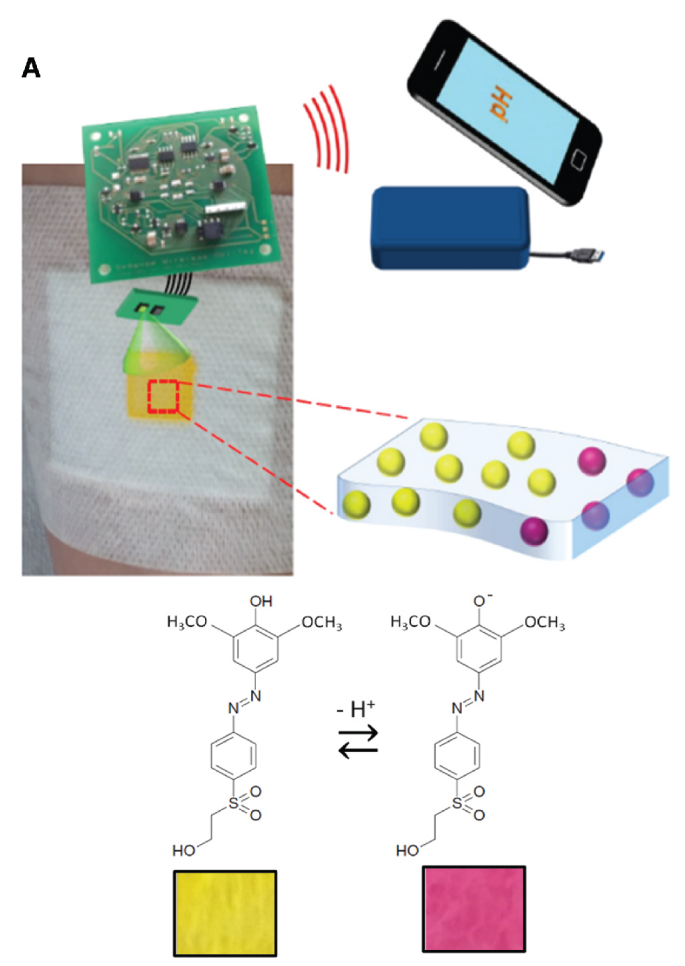

B
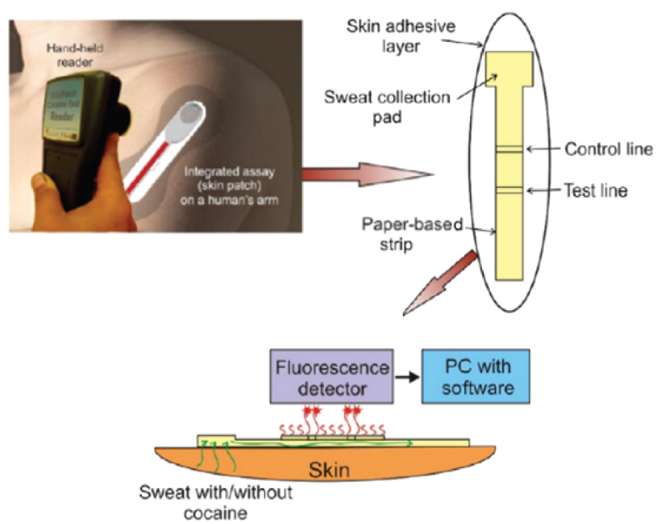

Figure 7: (A) Operation of the wireless smart bandage for optical sensing of wound $\mathrm{pH}$ (top); chemical structures of the protonated and deprotonated forms of the indicator dye with their respective colours (bottom) (Kassal et al. 2017). (B) Schematic visualisation of the microfluidic test strip with sample pad and a handheld fluorescence reader for detection of cocaine abuse (Walczak, Kruger \& Moynihan, 2015).

\subsubsection{Other applications}

Wearable sensors can also be used for in vivo monitoring with an "in-body wearable system," as demonstrated by Chan et al. (2015). Bio- or mucoadhesive anchoring is a challenge for the development of advanced gastrointestinal surgical instruments, endoluminal monitoring devices, and drug delivery systems. The authors presented a proof of concept of an endoluminal photoplethysmographic (PPG) sensing patch with bioadhesive polymers for the detection of gastrointestinal bleeding by direct optical absorption of LED light. The authors synthesised four different mucoadhesive films based on a biocompatible carbopol hydrogel. The PPG sensor was placed on a flexible circuit board and built in between two muchoadhesive films. The authors' findings demonstrate a potential for development of in vivo wearable systems. Wearable sensing goes a step further with using fingernails as a platform for sensors. Kim et al. (2016) have designed a colourimetric pH sensor on a polyvinyl chloride matrix in the shape of a fingernail. To expand the range of $\mathrm{pH}$ sensing, they used three different $\mathrm{pH}$ indicators: bromothymol blue, bromocresol green, and cresol red. The sensors provide reversible, repeatable, and fast response with an intense colour change. Such a wearable platform can be used for analysis of different samples. Duy et al. (2016) have developed a flexible and transparent chemical sensor for colourimetric and electrical sensing of ammonia gas (5-40 ppm). The sensor consists of reduced graphene oxide with $\mathrm{pH}$ indicator dye bromophenol blue on a PET substrate. When the sensor is exposed to ammonia gas, it visually changes colour depending on humidity conditions, and this can be detected using a smartphone camera. In contrast, the resistance of the device increases in the presence of ammonia and can be measured. Visual detection shows a very fast response time $(<15 \mathrm{~s})$ compared with electrical detection (15-20 min). However, visual mode has a limited working range and needs humid conditions.

Walczak, Kruger, and Moynihan (2015) have developed a test-strip fluorescent sensor for detection of cocaine in sweat. The lateral flow strip consists of a control line and test line, and recognition is based on a competitive immunoassay approach. A handheld fluorescence reader was used for concentration measurement (Figure 7B). Galpothdeniya et al. (2014) developed ionic liquid (IL)-based thread optoelectronic sensor arrays for $\mathrm{pH}$ detection in aqueous solutions. Twelve different sensors were fabricated by an ion exchange reaction between anion $\mathrm{pH}$ indicator dyes and cation trihexyl(tetradecyl)phosphonium. The synthesised IL showed low solubility in aqueous solutions. The authors immobilised IL sensors into four different matrices [glass microfiber filter papers, cotton thread, silica thin layer chromatography (TLC) plates, and alumina TLC plates] and used them for the colourimetric $\mathrm{pH}$ analysis of aqueous solutions and the detection of acidic and basic vapours. The 
results show that the alumina matrix is a good choice for acidic vapour sensing, and the silica matrix for basic vapour sensing. Thread-based sensor arrays have a great potential for application in the fabrication of wearable sensing because of its regeneration and reuse, as well as flexibility, small sample volume requirement, and low weight. Morales-Narváez et al. (2015) have reported on nanopaper-based platforms for sensors with plasmonic or photoluminescent properties. The platforms developed include a colourimetric sensor based on nanopaper containing embedded silver and gold nanoparticles, a photoluminescent sensor with CdSe@ZnS quantum dots conjugated to nanopaper, and a potential up-conversion sensing platform constructed from different nanoparticles. The authors indicate that the designed platforms can show high selectivity and specificity through functionalisation with biomolecules and they can work in complex matrixes.

\section{Smartphone camera-based optical sensors}

Today, the smartphone is near ubiquitous, and thanks to powerful processing capabilities, can function as both a readout and a data analysis unit, typically using standard radio protocols for readout such as Bluetooth, NFC, Wi-Fi, and GSM (Figure 1A). Meanwhile, the increasing quality of integrated cameras enables the smartphone to also serve as the detector in optical (bio)chemical sensing systems (Figure 1B).

Smartphones are an excellent mobile platform for optical sensing applications because of their integrated camera modules (focusing lens and CMOS sensor) and LED light illumination, as well as their ubiquity. The role of smartphone-based sensors and biosensors can be crucial in healthcare and environmental monitoring, security, or food quality control (Preechaburana, Suska \& Filippini, 2014; McCracken \& Yoon, 2016; Roda et al., 2016). In this section, we discuss chemical sensors and biosensors in which the optical signal is detected by smartphone-integrated physical sensors (almost exclusively the smartphone camera), as depicted in Figure 1B. The section is divided into three subsections based on key application areas of smartphone-based sensors - healthcare, environmental, and food quality monitoring. Of 77 publications covered by this review, 42 papers $(55 \%)$ were found to be smartphone-based optical sensors. The most commonly investigated group of smartphone-based optical sensors are systems designed for healthcare and point-of-care applications (43\%), then for environmental monitoring (40\%) and food quality (17\%).

Advancement in the use of the smartphone camera is probably most developed in smartphone-based fluorescence microscopy for imaging of various fluorescently labelled specimens in the field (Wei et al. 2013 and Contreras-Naranjo, Wei, and Ozcan 2016). Different smartphone microscopy setups have been reported, highlighting the potential for simplification and acceleration of many healthcare and point-of care applications. Another example of smartphone camera use is smartphone-based spectroscopy, which can easily replace expensive and bulky laboratory spectrometers. Recently reported smartphone spectrometers are mostly based on colourimetric (absorption or reflection) (Hu, Chen \& Sonkusale, 2015; Wang et al., 2016a), fluorescence (Yu, Tan \& Cunningham, 2014) or photonic crystal-based (Gallegos et al. 2013) detection. Several groups, however, presented smartphone sensing applications based on surface plasmon resonance (de Souza, Lima \& Neff, 2014; Guner et al., 2017) and successfully presented multichannel analyses (Wang et al. 2017).

\subsection{Healthcare and point-of-care diagnostics}

The most commonly investigated group of smartphone-based optical sensors are systems designed for healthcare and point-of-care applications (42.5\%) (Table 5). Adaptation of sensing systems for monitoring human health and application of such portable and integrated devices in medical diagnostics is extremely captivating. Telemedicine is rapidly advancing because of scientific studies on using smartphone functions in the development of novel accurate and sensitive diagnostic devices (Martinez et al. 2008). Built-in sensors, diverse applications, and constant connection through wireless data service make smartphones ideal point-of-care devices. Smartphone-based biosensing is demonstrated in different point-of-care diagnostic tests such as markers for HIV and hepatitis (Giavazzi et al. 2014), ovarian cancer biomarker (Wang et al. 2011), creatinine in urine samples (Debus et al. 2015), or bacteria in blood (Choi et al. 2016) and urine (Cho et al. 2015), showing that smartphonebased point-of-care diagnostics can become a broadly applied biotechnological tool. An easy-to-use point-ofcare device with a potential commercial application is demonstrated for the quantification of cholesterol (Oncescu, Mancuso \& Erickson, 2014), an alarming parameter important for preventing heart disease. Colourimetric quantification of cholesterol is enabled by enzymatic reactions on dry reagent strips. Reagents convert total cholesterol finally to quinoneimine dyes, which give the colourimetric response. 


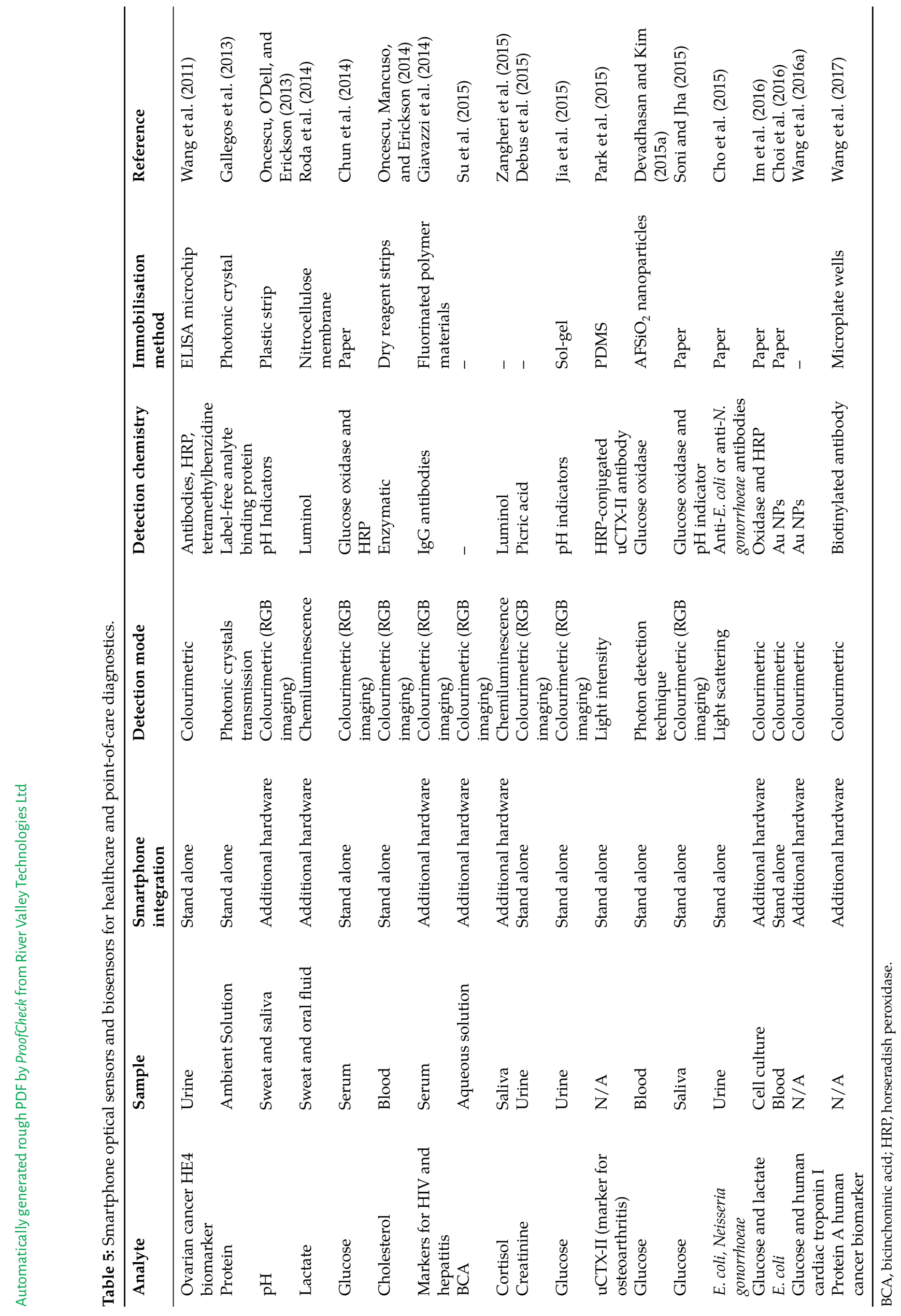


Smartphone optical sensors and biosensors for point-of-care applications mostly rely on embedded cameras for RGB imaging. However, there is an example of using other built-in sensors, such as the smartphone light meter, in biosensing of osteoarthritis marker (Park et al. 2015). The immunoblotting assay resulted in a precipitation reaction, which caused changes in light intensity. The integrated illumination sensor was used as a signal transducer, whereas changes in LED intensity passing through the sensing channel (polymer film) were numerically quantified using the lux meter application. Different techniques in smartphone optical sensing are presented in Figure 8.
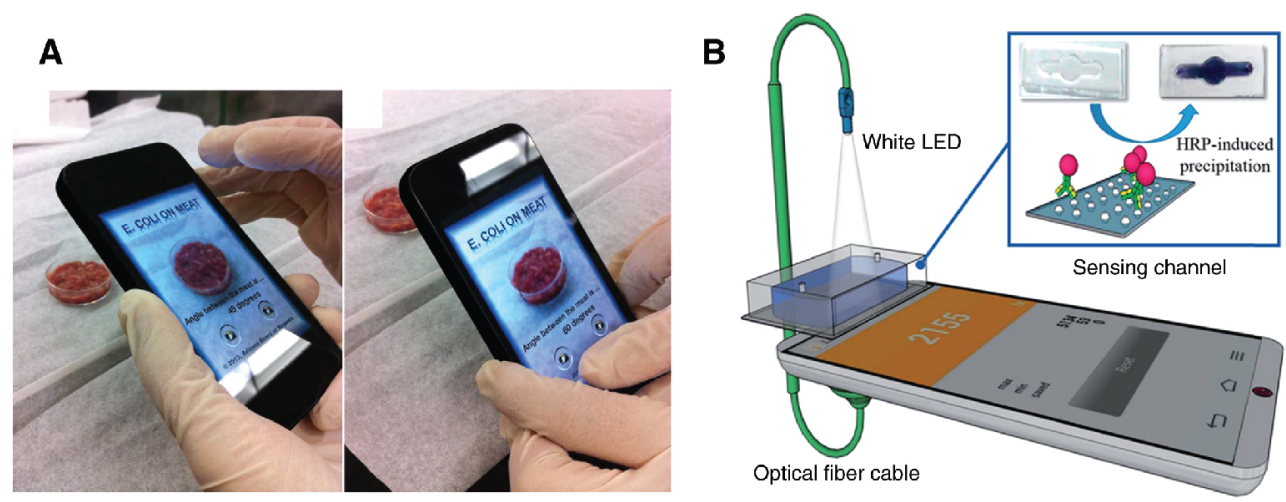

Figure 8: Different techniques in smartphone optical sensing.

(A) Optical sensing based on light scattering. Smartphone is applied for scatter detection of amines in rapid and reagentless detection of microbial contamination within meat (Liang, Park \& Yoon, 2014). (B) Optical sensing based on light intensity change caused by precipitation. Smartphone-based immunosensing system use built-in illumination sensor. The optical fiber was connected to the front of the smartphone to provide the LED flash. The intensity of LED light is directly dependent of concentration of osteoarthritis biomarker uCTX-II that was analysed by using the lux meter application (Park et al. 2015).

Amongst all point-of-care devices, glucose monitoring systems are the most widespread. The large number of diabetic patients and the need for self-monitoring blood glucose lead to the development of a large number of point-of-care glucose sensing devices. Unlike many electrochemical sensors, optical sensors for glucose determination are based on a noninvasive and painless mechanism of sampling and detection. Glucose sensing is adapted for smartphone detection by immobilising glucose oxidase, horseradish peroxidise, and/or a $\mathrm{pH}$-sensitive dye that would change colour due to gluconic acid formation during the enzymatic reaction. Smartphone optical sensors for glucose monitoring based on simple colourimetric imaging were developed for saliva (Soni and Jha 2015), urine (Jia et al. 2015), serum (Chun et al. 2014), or blood (Devadhasan and Kim 2015a). Glucose monitoring was also demonstrated by Wang et al. (2016b), presenting a smartphone-attached spectrometer. Performance of the smartphone spectrometer was compared with a commercial plate reader, revealing applicability in biosensing. Most suitable immobilisation methods presented for glucose determination are paper-based test strips (Chun et al. 2014, Soni and Jha 2015, and Im et al. 2016), sol-gel (Jia et al. 2015), or nanoparticles (Devadhasan et al. 2015b and Wang et al. 2016a).

Point-of-care diagnostics is mostly based on easy-to-use colourimetric analyses of disposable paper- or plastic-based tests to avoid an additional setup for complicated measurements. Optode-based sensors, commonly used in sensing almost all analytes, can provide a suitable sensing platform for smartphone-based optical sensors, as presented by Awqatty et al. (2014). Basic metabolic panel quantification, such as sodium, potassium, chloride, calcium, bicarbonate, glucose, urea, and creatinine, is demonstrated by smartphone fluorescence spectrophotometry. A smartphone attachment, in the form of a custom-made case consisting of a microcontroller and an optical chamber for fluorescent measurements, enables ratiometric measurements with minimal signal interference and inaccuracy. However, a smartphone accessory does not need to be expensive or robust, as presented by Zangheri et al. (2015), who demonstrated a simple 3D-printed smartphone attachment for the detection of salivary cortisol. The biosensor is based on a chemiluminescent lateral flow immunoassay method using peroxidase-cortisol conjugate and luminol/enhancer/hydrogen peroxide for detection. It should be mentioned that chemiluminescence measurements using a smartphone with minimum optical attachments are rare and challenging because of low signals that are often seriously interfered by background fluorescence or light scattering. However, even a smartphone-based bioluminescence whole-cell toxicity biosensor has been developed by Cevenini et al. (2016). 3D-printed cartridges were developed for bioluminescence detection based on greenemitting luciferase. Dimethyl sulfoxide was used as a model toxic compound on kidney cells, and a stand-alone platform for quantitative toxicity testing was presented.

Smartphone-based monitoring of athletes' performance via lactate detection in oral fluid and sweat is presented by Roda et al. (2014). They developed a chemiluminescent biosensor for sport and fitness applications 
based on lactate oxidase biosensing mechanism coupled with a luminol $/ \mathrm{H}_{2} \mathrm{O}_{2} /$ horseradish peroxidise enhancing system embedded in a 3D-printed smartphone accessory. Portable and noninvasive lactate measurement with a simple readout is one of rare examples of chemiluminescent smartphone sensors. The quantification of lactate lasted less than $5 \mathrm{~min}$ with detection limits of $0.1 \mathrm{mmol} / \mathrm{L}$ in sweat, which is comparable to wearable lactate sensors (Matzeu, Florea \& Diamond, 2015; Steinberg, Kassal \& Steinberg, 2016).

Sweat monitoring for athletes has also been demonstrated by Oncescu, O'Dell, and Erickson (2013) by developing an integrated smartphone accessory for $\mathrm{pH}$ control. Sweat $\mathrm{pH}$ can be correlated to sodium concentration and alarm the user to hydrate during physical exercise and avoid the risk of muscle cramps. The developed accessory is based on a slot containing disposable $\mathrm{pH}$ test strips for colourimetric analysis using a smartphone camera.

\subsection{Environmental monitoring}

Environmental issues with direct influence on healthcare and human well-being, such as water quality and bacterial outspread, are reaching alarming levels in some parts of the world. Smartphone-based optical sensors for environmental monitoring are the second most widespread group (40\%). Smartphone-based environmental monitoring is demonstrated in different matrices (soil, gas, water) and for diverse analytes ( $\mathrm{pH}$, chlorine, bacteria, heavy metals), as summarised in Table 6. Smartphone-based water quality control is presented via pH monitoring (Hossain et al. 2015a, 2015b), nitrite (Lopez-Ruiz et al. 2014), oxygen (Xu et al. 2015), catechols (Wang et al. 2016b), chlorine (Sumriddetchkajorn, Chaitavon \& Intaravanne 2013; 2014), or bacterial detection.

$\mathrm{pH}$ is one of the most important analytical parameters in environmental monitoring. Smartphone cameras are often used for measuring RGB intensities of pH indicators (Devadhasan and Kim 2015a and Pavai, Orosz, and Paszternak 2016). However, light intensity requirements, the difference between smartphone devices, or accuracy of measurements are often cited as main drawbacks (Xu et al. 2015 and Roda et al. 2016). To overcome reproducibility problems, $\mathrm{pH}$ indicators immobilised in hydrogel polyvinyl alcohol films are demonstrated as a reliable method for accurate smartphone-based real-time $\mathrm{pH}$ imaging (Devadhasan et al. 2015b). pH-sensitive hydrogel films also showed a potential for chemical vapour measurements.

Gunda et al. (2014) developed a portable water quality kit based on colourimetric biochemical detection of Escherichia coli. A smartphone accessory in the form of an external optical attachment is used for fluorescence detection of Salmonella and E. coli by a lateral flow immunoassay (Rajendran, Bakthavathsalam \& Ali, 2014). Bacterial detection on smartphone has also been demonstrated by a light scattering method on a ground beef sample (Liang, Park \& Yoon, 2014) and urine samples (Cho et al. 2015).

Low-cost and easy-to-use environmental monitoring in field and resource-limited settings is a global need. Monitoring of contamination with trace-level toxic heavy metal ions by smartphone is presented in water samples (Wei et al. 2014) and soil (Hu, Chen \& Sonkusale, 2015). Wei et al. demonstrated a smartphone-based quantification of mercury (II) ions in water samples with parts per billion level of sensitivity. A handheld platform attached to a smartphone detected mercury using a plasmonic gold nanoparticle and aptamer-based colourimetric transmission assay (Wei et al. 2014). This and other examples of opto-mechanical attachments for smartphone devices are demonstrated (Figure 9). 


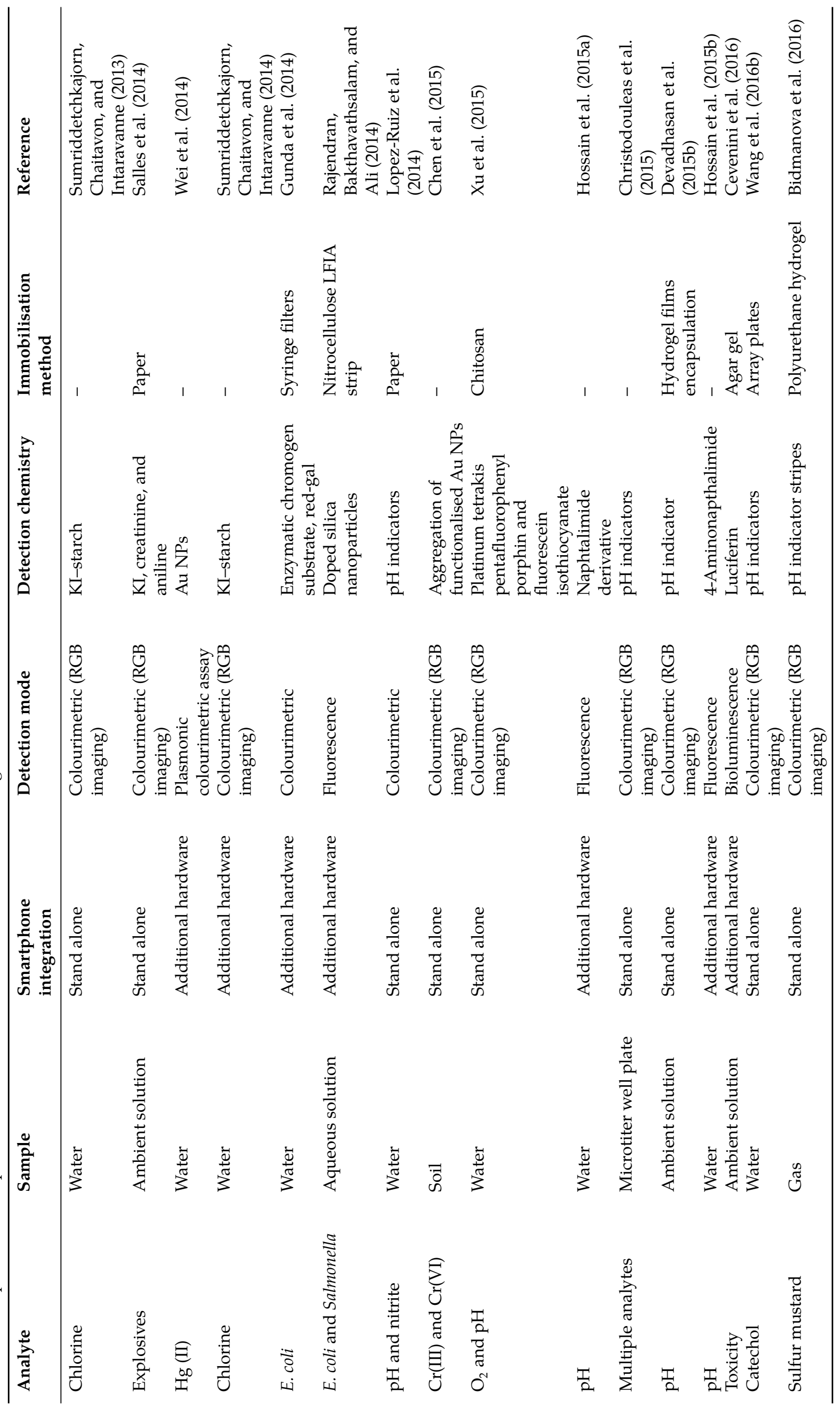


A

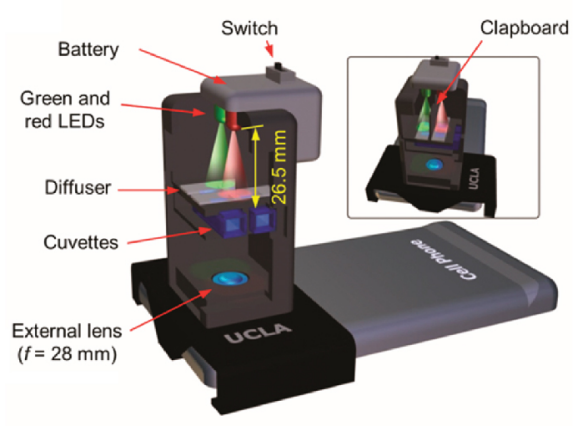

B

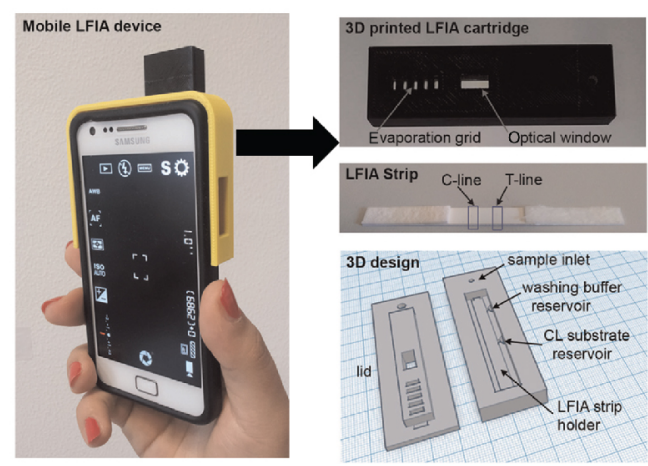

Figure 9: Opto-mechanical attachments for smartphone devices demonstrated as (A) ratiometric fluorescent detector for mercury contamination in water (Wei et al. 2014) and (B) integrated cortisol LFIA smartphone-based device (Zangheri et al. 2015).

Smartphone-based optical sensors can also offer fast detection of chemical agents of military and terroristic significance. Test strips for rapid detection of sulfur mustard by visual readout using enzyme haloalkane dehalogenase and $\mathrm{pH}$ indicators enable visual detection with high potential for smartphone applications (Bidmanova et al. 2016). A similar disposable paper-based platform is presented for colourimetric explosive discrimination (Salles et al. 2014).

\subsection{Food quality}

Smartphones are easily engaged in quality control processes in the food industry. Smartphone cameras can effectively replace robust and expensive instruments for colourimetric analysis and offer users low-cost and easy-to-use methods of monitoring and detection, especially in resource-limited environments. Easy-to-use smartphone-based detection of bacteria or amines attributed to food spoilage is demonstrated in several scientific publications presented in Table 7 (17.5\%). Optical chemical sensors in food quality control are widely applied. Integrating optical systems with smartphones for smart packaging applications (Bueno et al. 2015a) or test strips for food spoilage detection (Pavai, Orosz \& Paszternak, 2016) opens up new areas of research and potential new markets.

Colourimetric measurements are the most commonly used smartphone detection method, wherein microfluidic analysis presents some advantages over other chemical principles, because of the necessary miniaturisation of smartphone-based sensor systems. Park et al. (2014) presented a disposable sensor for red wine tasting based on mimicking the human tongue and nose (Figure 10). Paper microfluidics were used to detect taste chemicals whose colourimetric response in contact with chosen $\mathrm{pH}$ indicators is obtained by red, green, and blue pixel intensities and principal component analysis (Liang, Park \& Yoon, 2014). The authors successfully classified red wines by their sweetness and bodiness using a smartphone application. Wine quality can also be monitored through iron concentration, in which case colourimetric sensory solid polymer substrates for smartphone colourimetric detection of Fe(III) have been demonstrated (Vallejos et al. 2013). 


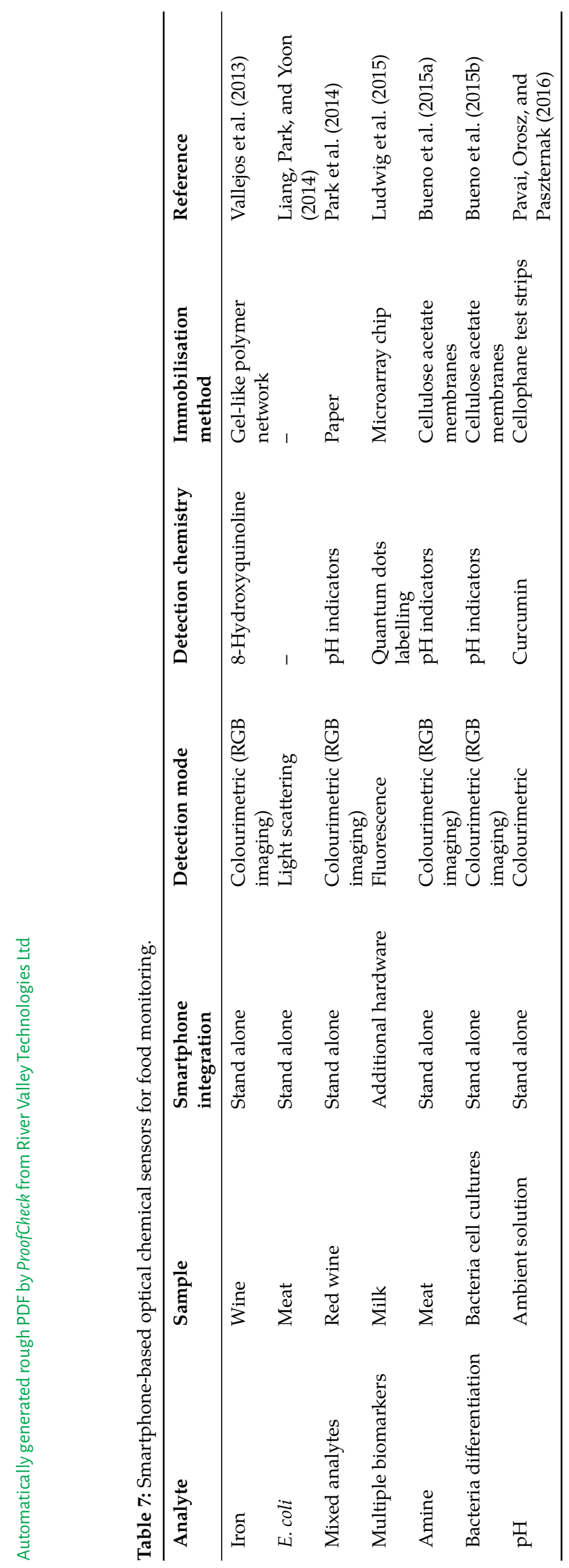


A

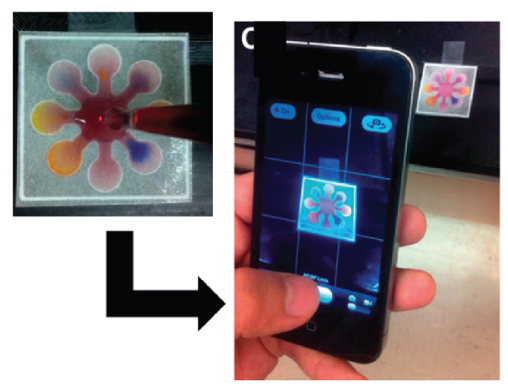

B

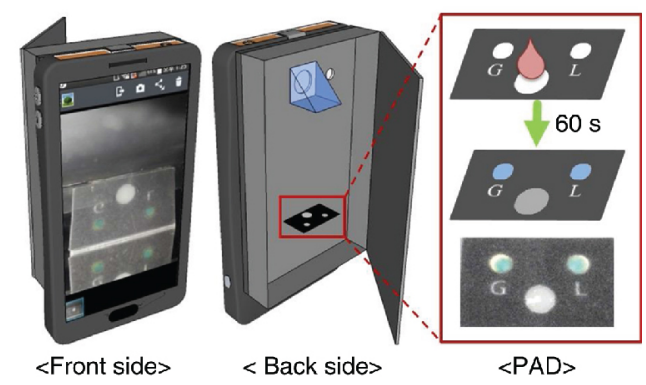

Figure 10: Smartphone-based colourimetric analysis on paper substrates: (A) RGB imaging of paper-based microfluidic chip for red wine tasting (Park et al. 2014); (B) Paper-based analytical device (PAD) for monitoring glucose and lactate concentrations in the cell culture medium (Im et al. 2016). The PAD image is observed by trigonal prism and smartphoneembedded camera.

Colourimetric sensors mostly use built-in smartphone sensors. However, if analyte detection is based on a fluorescence signal, the smartphone device often needs an extra optical component such as light source or filters. To obtain a low-cost and low-weight smartphone accessory for fluorescence detection, Ludwig et al. (2015) developed a 3D-printed opto-mechanical attachment for detection of multiple biomarkers in milk extracts. Multiple immunoassays were designed on a microarray chip followed by labelling 48 microspots with quantum dots. QD fluorescence excited by UV light from LEDs embedded in the smartphone attachment was detected by the smartphone camera.

\section{Findings of the review}

Wireless and mobile optical chemical sensors and biosensors, using different modes of optical sensing - measuring absorbance, reflectance, fluorescence, chemiluminescence, refractive index, and surface plasmon resonance - are being developed through academic research. These sensors are targeted at four main application areas: healthcare and medicine (38\%), environmental monitoring (31\%), food quality (11\%), and sport and fitness (7\%) (Figure 11A). The field has seen large growth in publication productivity over several consecutive years (Figure 2) partly because of the increased popularity of smartphone-based systems and wearable systems and partly because of the increased demand for rapid point-of-measurement analytical chemistry.
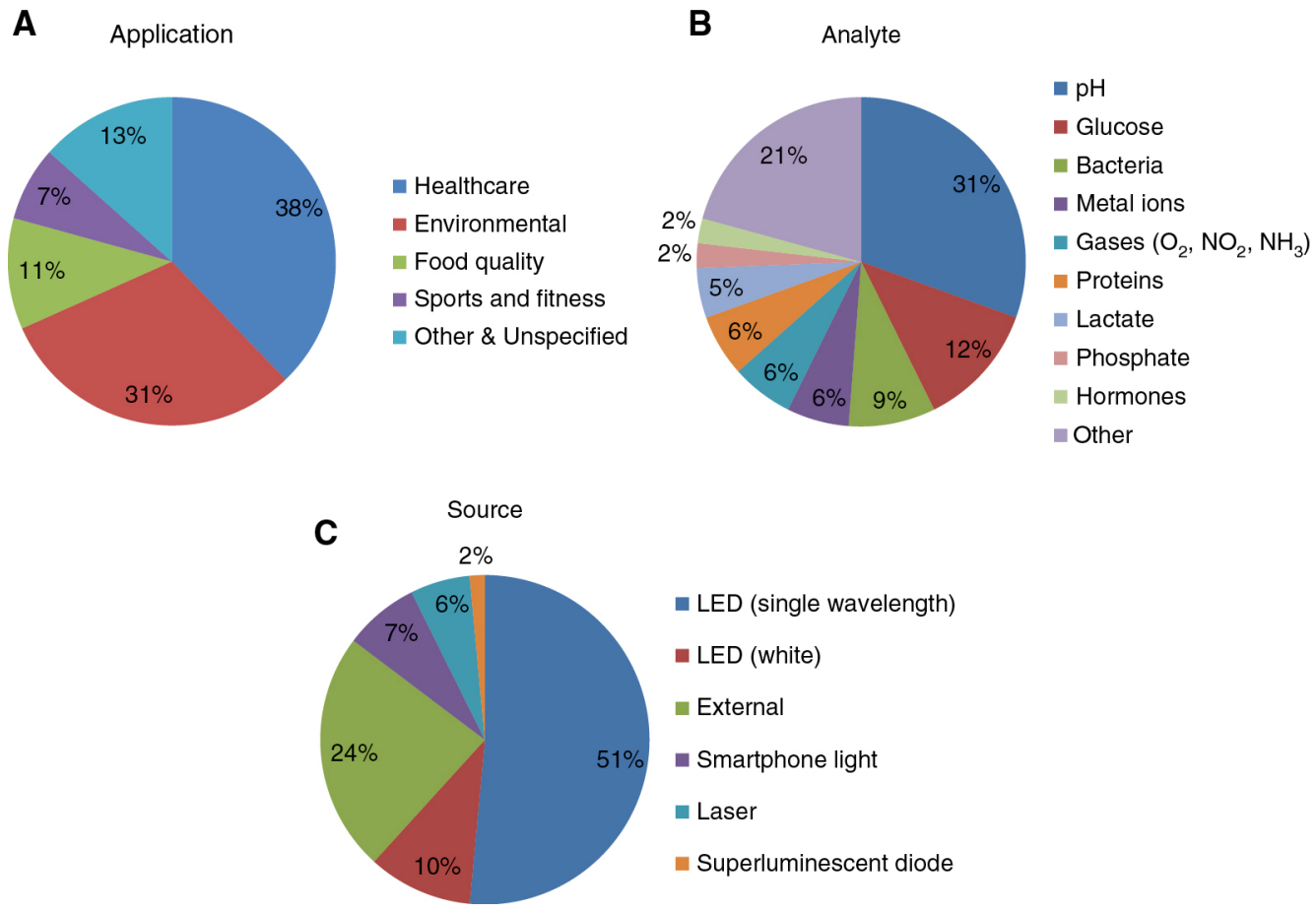

- LED (single wavelength)

- LED (white)

External

- Smartphone light

- Laser

- Superluminescent diode

Figure 11: Distribution of wireless and mobile optical chemical sensors and biosensors by (A) application, (B) analyte, and $(\mathrm{C})$ light source. 
Across the three groups of sensor classes we categorised here (wireless, wearable, and smartphone camerabased), $\mathrm{pH}$ is the most common target analyte (Figure 11B). Although $\mathrm{pH}$ is clearly an important analyte for many application areas ( $\mathrm{pH}$ of wound fluid and sweat, $\mathrm{pH}$ of aquatic environments, air acidity, etc.), the other reason for this could be the abundance of different $\mathrm{pH}$-sensitive chromophores and fluorophores that can easily be incorporated into solid-state optical sensors. Other popular analytes include glucose, bacteria, metal ions, gases $\left(\mathrm{O}_{2}, \mathrm{NO}_{2}\right.$, and $\left.\mathrm{NH}_{3}\right)$, proteins, and lactate.

Power consumption of wireless sensors is one of the main issues for their successful implementation. The most power-hungry elements in an optical chemical sensor are the light source and wireless transceiver. Consequently, the most popular light sources are the ones that consume the least power - LEDs. There are examples of sensors using laser sources and superluminescent diodes, but $61 \%$ of the sensors identified in our survey use an LED source (Figure 11C). Although some colourimetric sensors use a white LED and RGB readout, detection with a single-wavelength LED and suitable photodetector is more common, most likely because of the simpler data analysis. Many sensors (mainly smartphone-based) rely on external light sources, such as ambient light, lamps, or even light from image scanners.

Smartphones themselves are an attractive platform for the development of optical sensors and biosensors, but the achievement of satisfactory analytical performance is often challenging. In the simplest form of smartphone-based sensing systems, optical signals are detected by the integrated camera in the form of an image, which is then further analysed by an analytical software running on the phone. This approach generally does not require any extra hardware attachments or accessories. However, major drawbacks such as interference of ambient light, differences between smartphone devices, and semiquantitative analyses must be overcome. It is important to mention that differences between smartphones and their integrated cameras and the hardware and low-level software used to control them are highly significant. Images acquired by smartphone camera need to look natural to the human eye, which does not necessarily mean that they are entirely reliable or accurate at differentiating true red, green, and blue colours. Because of, for example, "autofocus," "autoexposure," and "white balance" corrections, it is often difficult to reproducibly recognise the "tones" of a certain colour (McCracken and Yoon 2016). The optical attachments are often complex and large in size, especially attachments for fluorescence measurements because of the need for extra optical components. Approximately $38 \%$ of the smartphone-based systems identified in our review incorporated some form of additional hardware to improve the optical signal detection. Nevertheless, colourimetric measurement of biochemical test strips by smartphone has shown potential for widespread use. Such point-of-care tests can be carried out without any extra attachments, outside of laboratory, and under ambient light.

Wearable optical sensors are being developed mainly on textile, paper, and polymer substrates to ensure flexibility and comfort for the end user. The focus of the research teams developing such sensors has clearly been on sweat and wound fluid analysis.

\section{Conclusions and outlook}

The choice of chemical sensor architecture (sensing scheme, materials, and immobilisation method) and wireless technology are application-dependent because they affect multiple parameters, such as sensitivity, limit of detection, power consumption, battery lifetime, read range, data rate, and so on. Several of the analysed wireless sensors used proprietary wireless modules, but the research focus has shifted towards standard wireless communication, namely, Bluetooth, ZigBee, RFID, and NFC. Because of the pervasiveness of smartphones, smartphone-compatible radio standards, such as Bluetooth and NFC, are becoming increasingly popular as the platform of choice for optical chemical sensor researchers.

In the future, we expect a tighter integration of the chemical sensor with miniaturised wearable platforms providing sample interface, light source, detector, data storage, processing, and radio communication, similar to the trend apparent in the wearable electrochemical sensor field. Flexible and stretchable wearable electronics, as well as advances in flexible light sources and photodetectors, could facilitate such integration. Ultimately, smartphone-integrated wireless communication could be used to retrieve analytical data from a wearable optical sensor, thus retaining complete mobility of the system while avoiding the problems associated with smartphone camera readout.

\section{References}

Awqatty, B.; Samaddar, S.; Cash, K. J.; Clark, H. A.; Dubach, J. M. Fluorescent sensors for the basic metabolic panel enable measurement with a smart phone device over the physiological range. Analyst 2014, 139, 5230-5238. 
Bandodkar, A. J.; Wang, J. Non-invasive wearable electrochemical sensors: a review. Trends Biotechnol. 2014, 32, 363-371.

Baysal, G.; Kok, F. N.; Trabzon, L.; Kizil, H.; Cocek, I.; Kayaoglu, B. K. Microfluidic nonwoven-based device as a potential biosensor for sweat analysis. Mechanical Design and Power Engineering. 2014, 490-491, 274-279.

Bidmanova, S.; Steiner, M. S.; Stepen, M.; Vymazalova, K.; Cruber, M. A.; Duerkop, A.; Damborsky, J.; Prokop, Z.; Wolfbeis, O. S. Enzyme-based test strips for visual or photographic detection and quantitation of gaseous sulfur mustard. Anal. Chem. 2016, 88, 6044-6049.

Bueno, L.; Cottell, A.; Reddy, S. M.; Paixao, T. Coupling dye-integrated polymeric membranes with smartphone detection to classify bacteria. Rsc Adv. 2015a, 5, 97962-97965.

Bueno, L.; Meloni, G. N.; Reddy, S. M.; Paixao, T. Use of plastic-based analytical device, smartphone and chemometric tools to discriminate amines. Rsc Adv. 2015b, 5, 20148-20154.

Caldara, M.; Colleoni, C.; Guido, E.; Re, V.; Rosace, G. Development of a textile-optoelectronic pH meter based on hybrid xerogel doped with methyl red. Sensors Actuators B Chem. 2012, 171, 1013-1021.

Caldara, M.; Colleoni, C.; Cuido, E.; Re, V.; Rosace, C. Optical monitoring of sweat pH by a textile fabric wearable sensor based on covalently bonded litmus-3-glycidoxypropyltrimethoxysilane coating. Sensors Actuators B Chem. 2016, 222, 213-220.

Camarillo-Escobedo, R. M.; Valdés-Perezgasga, F.; Rodríguez-Rivera, R. Micro-analyzer with optical detection and wireless communications. Sensors Actuators A Phys. 2013, 199, 181-186.

Cao, F.; Wang, Y.-D.; Liu, L.; Cong, M.-L. A wireless sensor network based on DFB lasers for water vapor detection. Sensors Actuators B Chem. 2014, 193, 370-374.

Capitán-Vallvey, L. F.; Palma, A. J. Recent developments in handheld and portable optosensing - a review. Anal. Chim. Acta. 2011, 696, 27-46.

Capitán-Vallvey, L. F.; López-Ruiz, N.; Martínez-Olmos, A.; Erenas, M. M.; Palma, A. J. Recent developments in computer vision-based analytical chemistry: a tutorial review. Anal. Chim. Acta. 2015, 899, 23-56.

Cevenini, L.; Calabretta, M. M.; Tarantino, G.; Michelini, E.; Roda, A. Smartphone-interfaced 3D printed toxicity biosensor integrating bioluminescent "sentinel cells". Sensors Actuators B Chem. 2016, 225, 249-257.

Chan, C. K. W.; Zheng, Y. L.; Siu, E. H. L.; Yu, R. X.; Leung, B. H. K.; Zhang, R.; Poon, C. C. Y. A mucoadhesive endoluminal wearable sensory system. 2015 37th Annual International Conference of the IEEE Engineering in Medicine and Biology Society, 2015, pp 4355-4358.

Chen, W.; Cao, F.; Zheng, W.; Tian, Y.; Xianyu, Y.; Xu, P.; Zhang, W.; Wang, Z.; Deng, K.; Jiang, X. Detection of the nanomolar level of total $\operatorname{Cr}[(i i i)$ and (vi)] by functionalized gold nanoparticles and a smartphone with the assistance of theoretical calculation models. Nanoscale. 2015, 7, 2042-2049.

Cho, S.; Park, T. S.; Nahapetian, T. G.; Yoon, J. Y. Smartphone-based, sensitive RAD detection of urinary tract infection and gonorrhea. Biosens. Bioelectron. 2015, 74, 601-611.

Choi, S. Powering point-of-care diagnostic devices. Biotechnol. Adv. 2016, 34, 321-330.

Choi, J. R.; Hu, J.; Tang, R.; Cong, Y.; Feng, S.; Ren, H.; Wen, T.; Li, X.; Wan Abas, W. A. B.; Pingguan-Murphy, B.; Xu, F. An integrated paperbased sample-to-answer biosensor for nucleic acid testing at the point of care. Lab Chip 2016, 16, 611-621.

Christodouleas, D. C.; Nemiroski, A.; Kumar, A. A.; Whitesides, G. M. Broadly available imaging devices enable high-quality low-cost photometry. Anal. Chem. 2015, 87, 9170-9178.

Chun, H. J.; Park, Y. M.; Han, Y. D.; Jang, Y. H.; Yoon, H. C. Paper-based glucose biosensing system utilizing a smartphone as a signal reader. Biochip]. 2014, 8, 218-226.

Contreras-Naranjo, J. C.; Wei, Q. S.; Ozcan, A. Mobile phone-based microscopy, sensing, and diagnostics. IEEEJ. Sel. Top. Quantum Electron. 2016, 22, 14 .

Curto, V. F.; Coyle, S.; Byrne, R.; Angelov, N.; Diamond, D.; Benito-Lopez, F. Concept and development of an autonomous wearable microfluidic platform for real time pH sweat analysis. Sensors Actuators B Chem. 2012, 175, 263-270.

Czugala, M.; Gorkin, R., III; Phelan, T.; Gaughran, J.; Curto, V. F.; Ducree, J.; Diamond, D.; Benito-Lopez, F. Optical sensing system based on wireless paired emitter detector diode device and ionogels for lab-on-a-disc water quality analysis. Lab Chip 2012, 12, 5069-5078.

de Souza, C. A.; Lima, A. M. N.; Neff, H. Smartphone based, portable optical biosensor utilizing surface plasmon resonance. 2014 IEEE International Instrumentation and Measurement Technology Conference (I $\mathrm{mtC}$ ) Proceedings. 2014, 890-895.

Debus, B.; Kirsanov, D.; Yaroshenko, I.; Sidorova, A.; Piven, A.; Legin, A. Two low-cost digital camera-based platforms for quantitative creatinine analysis in urine. Anal. Chim. Acta. 2015, 895, 71-79.

Deng, S. J.; Doherty, W.; McAuliffe, M. A. P.; Salaj-Kosla, U.; Lewis, L.; Huyet, G. A low-cost, portable optical sensing system with wireless communication compatible of real-time and remote detection of dissolved ammonia. Photonic Sens. 2016, 6, 107-114.

Devadhasan, J. P.; Kim, S. An ultrasensitive method of real time $\mathrm{pH}$ monitoring with complementary metal oxide semiconductor image sensor. Anal. Chim. Acta. 2015a, 858, 55-59.

Devadhasan, J. P.; Oh, H.; Choi, C. S.; Kim, S. Whole blood glucose analysis based on smartphone camera module. J. Biomed. Opt. $2015 \mathbf{b}, 20,10$.

Diamond, D.; Coyle, S.; Scarmagnani, S.; Hayes, J. Wireless sensor networks and chemo-/biosensing. Chem. Rev. 2008, 108, 652-679.

Duy, L. T.; Trung, T. Q.; Dang, V. Q.; Hwang, B. U.; Siddiqui, S.; Son, I. Y.; Yoon, S. K.; Chung, D. J.; Lee, N. E. Flexible transparent reduced graphene oxide sensor coupled with organic dye molecules for rapid dual-mode ammonia gas detection. Adv. Funct. Mater. 2016, 26, 4329-4338.

Farre, M.; Kantiani, L.; Perez, S.; Barcelo, D. Sensors and biosensors in support of EU Directives. Trac-Trends Anal. Chem. 2009, 28, 170-185.

Fay, C.; Lau, K. T.; Beirne, S.; Conaire, C. O.; McGuinness, K.; Corcoran, B.; O'Connor, N. E.; Diamond, D.; McGovern, S.; Coleman, G.; Shepherd, R.; Alici, G.; Spinks, G.; Wallace, G. Wireless aquatic navigator for detection and analysis (WANDA). Sensors Actuators B Chem. 2010, 150, $425-435$.

Gallegos, D.; Long, K. D.; Yu, H. J.; Clark, P. P.; Lin, Y. X.; Ceorge, S.; Nath, P.; Cunningham, B. T. Label-free biodetection using a smartphone. Lab Chip 2013, 13, 2124-2132.

Galpothdeniya, W. I. S.; McCarter, K. S.; De Rooy, S. L.; Regmi, B. P.; Das, S.; Hasan, F.; Tagge, A.; Warner, I. M. lonic liquid-based optoelectronic sensor arrays for chemical detection. Rsc Adv. 2014, 4, 7225-7234.

Chafar-Zadeh, E. Wireless integrated biosensors for point-of-care diagnostic applications. Sensors (Basel, Switzerland). 2015, 15, 3236-3261. 
Giavazzi, F.; Salina, M.; Ceccarello, E.; Ilacqua, A.; Damin, F.; Sola, L.; Chiari, M.; Chini, B.; Cerbino, R.; Bellini, T.; Buscaglia, M. A fast and simple label-free immunoassay based on a smartphone. Biosens. Bioelectron. 2014, 58, 395-402.

Gunda, N. S. K.; Naicker, S.; Shinde, S.; Kimbahune, S.; Shrivastava, S.; Mitra, S. Mobile Water Kit (MWK): a smartphone compatible low-cost water monitoring system for rapid detection of total coliform and E. coli. Anal. Methods. 2014, 6, 6236-6246.

Guner, H.; Ozgur, E.; Kokturk, G.; Celik, M.; Esen, E.; Topal, A. E.; Ayas, S.; Uludag, Y.; Elbuken, C.; Dana, A. A smartphone based surface plasmon resonance imaging (SPRi) platform for on-site biodetection. Sensors Actuators B Chem. 2017, 239, 571-577.

Hossain, M. A.; Canning, J.; Ast, S.; Rutledge, P. J.; Jamalipour, A. Early warning smartphone diagnostics for water security and analysis using real-time $\mathrm{pH}$ mapping. Photon. Sens. 2015a, 5, 289-297.

Hossain, M. A.; Canning, J.; Ast, S.; Rutledge, P. J.; Yen, T. L.; Jamalipour, A. Lab-in-a-phone: smartphone-based portable fluorometer for pH measurements of environmental water. IEEE Sens. J. 2015b, 15, 5095-5102.

$\mathrm{Hu}, \mathrm{P.} \mathrm{X.;} \mathrm{Chen,} \mathrm{Y.;} \mathrm{Sonkusale,} \mathrm{S.} \mathrm{Low} \mathrm{cost} \mathrm{spectrometer} \mathrm{accessory} \mathrm{for} \mathrm{cell} \mathrm{phone} \mathrm{based} \mathrm{optical} \mathrm{sensor.} \mathrm{In} 2015$ Virtual Conference on Application of Commercial Sensors (pp. 1-5). New York: Curran Associates, Inc., 2015.

Huang, X.; Liu, Y. H.; Chen, K. L.; Shin, W. J.; Lu, C. J.; Kong, G. W.; Patnaik, D.; Lee, S. H.; Cortes, J. F.; Rogers, J. A. Stretchable, wireless sensors and functional substrates for epidermal characterization of sweat. Small 2014, 10, 3083-3090.

Im, S. H.; Kim, K. R.; Park, Y. M.; Yoon, J. H.; Hong, J. W.; Yoon, H. C. An animal cell culture monitoring system using a smartphone-mountable paper-based analytical device. Sensors Actuators B Chem. 2016, 229, 166-173.

ITU. Measuring the Information Society Report 2016. International Telecommunication Union, Ceneva Switzerland. 2016.

Jia, M. Y.; Wu, Q. S.; Li, H.; Zhang, Y.; Cuan, Y. F.; Feng, L. The calibration of cellphone camera-based colorimetric sensor array and its application in the determination of glucose in urine. Biosens. Bioelectron. 2015, 74, 1029-1037.

Kassal, P.; Zubak, M.; Scheipl, G.; Mohr, G. J.; Steinberg, M. D.; Steinberg, I. M. Smart bandage with wireless connectivity for optical monitoring of pH. Sensors Actuators B Chem. 2017, 246, 455-460.

Kassal, P.; Steinberg, M. D.; Steinberg, I. M. Wireless chemical sensors and biosensors: a review. Sensors Actuators B Chem. 2018, 266, $228-245$.

Kim, J.; Cho, T. N.; Valdes-Ramirez, G.; Wang, J. A wearable fingernail chemical sensing platform: pH sensing at your fingertips. Talanta 2016, $150,622-628$.

Koh, A.; Kang, D.; Xue, Y.; Lee, S.; Pielak, R. M.; Kim, J.; Hwang, T.; Min, S.; Banks, A.; Bastien, P.; Manco, M. C.; Wang, L.; Ammann, K. R.; Jang, K. I.; Won, P.; Han, S.; Chaffari, R.; Paik, U.; Slepian, M. J.; Balooch, G.; Huang, Y. G.; Rogers, J. A. A soft, wearable microfluidic device for the capture, storage, and colorimetric sensing of sweat. Sci. Transl. Med. 2016, 8, 13

Liang, P. S.; Park, T. S.; Yoon, J. Y. Rapid and reagentless detection of microbial contamination within meat utilizing a smartphone-based biosensor. Sci. Rep. 2014, 4, 5953.

Llorente-Alonso, A.; Pena-Poza, J.; de Arcas, G.; Garcia-Heras, M.; Lopez, J. M.; Villegas, M. A. Interface electronic system for measuring air acidity with optical sensors. Sensors Actuators A Phys. 2013, 194, 67-74.

Lopez-Ruiz, N.; Curto, V. F.; Erenas, M. M.; Benito-Lopez, F.; Diamond, D.; Palma, A. J.; Capitan-Vallvey, L. F. Smartphone-based simultaneous ph and nitrite colorimetric determination for paper microfluidic devices. Anal. Chem. 2014, 86, 9554-9562.

Ludwig, S. K. J.; Tokarski, C.; Lang, S. N.; van Cinkel, L. A.; Zhu, H.; Ozcan, A.; Nielen, M. W. F. Calling biomarkers in milk using a protein microarray on your smartphone. PLoS One 2015, 10, e0134360.

Malon Radha, S. P.; Heng Lee, Y.; Córcoles Emma, P. Recent developments in microfluidic paper-, cloth-, and thread-based electrochemical devices for analytical chemistry. Rev. Anal. Chem. 2017, 36, (4). 20160018.

Martinez, A. W.; Phillips, S. T.; Carrilho, E.; Thomas, S. W.; Sindi, H.; Whitesides, G. M. Simple telemedicine for developing regions: camera phones and paper-based microfluidic devices for real-time, off-site diagnosis. Anal. Chem. 2008, 80, 3699-3707.

Martinez-Olmos, A.; Fernandez-Salmeron, J.; Lopez-Ruiz, N.; Rivadeneyra Torres, A.; Capitan-Vallvey, L. F.; Palma, A. J. Screen printed flexible radiofrequency identification tag for oxygen monitoring. Anal. Chem. 2013, 85, 11098-11105.

Maruo, Y. Y.; Nakamura, M.; Higashijima, Y.; Kikuya, Y.; Nakamura, M. Development of highly sensitive nitrogen dioxide monitoring device and its application to wide-area ubiquitous network. Sensors Actuators B Chem. 2012, 173, 191-196.

Matzeu, G.; Florea, L.; Diamond, D. Advances in wearable chemical sensor design for monitoring biological fluids. Sensors Actuators B Chem. 2015, 211, 403-418.

McCracken, K. E.; Yoon, J.-Y. Recent approaches for optical smartphone sensing in resource-limited settings: a brief review. Anal. Methods 2016, 8, 6591-6601.

Mehmood, N.; Hariz, A.; Fitridge, R.; Voelcker, N. H. Applications of modern sensors and wireless technology in effective wound management. J. Biomed. Mater. Res. Part B Appl Biomater. 2014, 102, 885-895.

Morales-Narváez, E.; Colmohammadi, H.; Naghdi, T.; Yousefi, H.; Kostiv, U.; Horak, D.; Pourreza, N.; Merkoci, A. Nanopaper as an optical sensing platform. Acs Nano. 2015, 9, 7296-7305.

Morris, D.; Coyle, S.; Wu, Y. Z.; Lau, K. T.; Wallace, G.; Diamond, D. Bio-sensing textile based patch with integrated optical detection system for sweat monitoring. Sensors Actuators B Chem. 2009, 139, 231-236.

Mortellaro, M.; DeHennis, A. Performance characterization of an abiotic and fluorescent-based continuous glucose monitoring system in patients with type 1 diabetes. Biosens. Bioelectron. 2014, 61, 227-231.

Nemiroski, A.; Ryou, M.; Thompson, C. C.; Westervelt, R. M. Swallowable fluorometric capsule for wireless triage of gastrointestinal bleeding. Lab Chip 2015, 15, 4479-4487.

Oncescu, V.; O'Dell, D.; Erickson, D. Smartphone based health accessory for colorimetric detection of biomarkers in sweat and saliva. Lab Chip 2013, 13, 3232-3238.

Oncescu, V.; Mancuso, M.; Erickson, D. Cholesterol testing on a smartphone. Lab Chip 2014, 14, 759-763.

Pang, C.; Yu, M.; Zhang, X. M.; Cupta, A. K.; Bryden, K. M. Multifunctional optical MEMS sensor platform with heterogeneous fiber optic Fabry-Perot sensors for wireless sensor networks. Sensors Actuators A Phys. 2012, 188, 471-480.

Park, T. S.; Baynes, C.; Cho, S. I.; Yoon, J. Y. Paper microfluidics for red wine tasting. Rsc Adv. 2014, 4, 24356-24362.

Park, Y. M.; Han, Y. D.; Kim, K. R.; Zhang, C.; Yoon, H. C. An immunoblot-based optical biosensor for screening of osteoarthritis using a smartphone-embedded illuminometer. Anal. Methods 2015, 7, 6437-6442. 
Pasche, S.; Angeloni, S.; Ischer, R.; Liley, M.; Lupranoe, J.; Voirin, G. Wearable Biosensors for Monitoring Wound Healing. In Biomedical Applications of Smart Materials, Nanotechnology and Micro/Nano Engineering. P. Vincenzini and D. DeRossi Eds.; 2009; Vol. 57; pp 80-87.

Pavai, M.; Orosz, E.; Paszternak, A. Smartphone-based extension of the curcumin/cellophane pH sensing method. Food Anal. Methods. 2016, 9 , 1046-1052.

Potyrailo, R. A.; Surman, C.; Nagraj, N.; Burns, A. Materials and transducers toward selective wireless gas sensing. Chem. Rev. 2011, 111, 73157354.

Preechaburana, P.; Suska, A.; Filippini, D. Biosensing with cell phones. Trends Biotechnol. 2014, 32, 351-355.

Rajendran, V. K.; Bakthavathsalam, P.; Ali, B. M. J. Smartphone based bacterial detection using biofunctionalized fluorescent nanoparticles. Microchimica Acta. 2014, 181, 1815-1821.

Roda, A.; Guardigli, M.; Calabria, D.; Calabretta, M. M.; Cevenini, L.; Michelini, E. A 3D-printed device for a smartphone-based chemiluminescence biosensor for lactate in oral fluid and sweat. Analyst 2014, 139, 6494-6501.

Roda, A.; Michelini, E.; Zangheri, M.; Di Fusco, M.; Calabria, D.; Simoni, P. Smartphone-based biosensors: a critical review and perspectives. TrAC Trends Anal. Chem. 2016, 79, 317-325.

Salles, M. O.; Meloni, G. N.; de Araujo, W. R.; Paixao, T. Explosive colorimetric discrimination using a smartphone, paper device and chemometrical approach. Anal. Methods 2014, 6, 2047-2052.

Schyrr, B.; Pasche, S.; Scolan, E.; Ischer, R.; Ferrario, D.; Porchet, J. A.; Voirin, G. Development of a polymer optical fiber pH sensor for on-body monitoring application. Sensors Actuators B Chem. 2014, 194, 238-248.

Sequeira, M.; Bowden, M.; Minogue, E.; Diamond, D. Towards autonomous environmental monitoring systems. Talanta 2002, 56, 355-363.

Shepherd, R.; Beirne, S.; Lau, K. T.; Corcoran, B.; Diamond, D. Monitoring chemical plumes in an environmental sensing chamber with a wireless chemical sensor network. Sensors Actuators B Chem. 2007, 121, 142-149.

Slater, C.; Cleary, J.; McGraw, C. M.; Yerazunis, W. S.; Lau, K. T.; Diamond, D. Autonomous field-deployable device for the measurement of phosphate in natural water. Advanced Environmental, Chemical, and Biological Sensing Technologies V. T. VoDinh, R. A. Lieberman and G. Gauglitz, 2007; 6755.

Soni, A.; Jha, S. K. A paper strip based non-invasive glucose biosensor for salivary analysis. Biosens. Bioelectron. 2015, 67, 763-768.

Steinberg, I. M.; Steinberg, M. D. Radio-frequency tag with optoelectronic interface for distributed wireless chemical and biological sensor applications. Sensors Actuators B Chem. 2009, 138, 120-125.

Steinberg, M. D.; Kassal, P.; Tkalcec, B.; Steinberg, I. M. Miniaturised wireless smart tag for optical chemical analysis applications. Talanta 2014, 118, 375-381.

Steinberg, M. D.; Kassal, P.; Steinberg, I. M. System architectures in wearable electrochemical sensors. Electroanalysis 2016, 28, 1149-1169.

Su, K.; Zou, Q.; Zhou, J.; Zou, L.; Li, H.; Wang, T.; Hu, N.; Wang, P. High-sensitive and high-efficient biochemical analysis method using a bionic electronic eye in combination with a smartphone-based colorimetric reader system. Sensors Actuators B Chem. 2015, 216, 134-140.

Sumriddetchkajorn, S.; Chaitavon, K.; Intaravanne, Y. Mobile device-based self-referencing colorimeter for monitoring chlorine concentration in water. Sensors Actuators B Chem. 2013, 182, 592-597.

Sumriddetchkajorn, S.; Chaitavon, K.; Intaravanne, Y. Mobile-platform based colorimeter for monitoring chlorine concentration in water. Sensors Actuators B Chem. 2014, 191, 561-566.

Tamayol, A.; Akbari, M.; Zilberman, Y.; Comotto, M.; Lesha, E.; Serex, L.; Bagherifard, S.; Chen, Y.; Fu, G. Q.; Ameri, S. K.; Ruan, W. T.; Miller, E. L.; Dokmeci, M. R.; Sonkusale, S.; Khademhosseini, A. Flexible pH-sensing hydrogel fibers for epidermal applications. Adv Healthcare Mater. 2016, 5, 711-719.

Valdastri, P.; Susilo, E.; Foerster, T.; Strohhoefer, C.; Menciassi, A.; Dario, P. Wireless implantable electronic platform for chronic fluorescentbased biosensors. IEEE Trans. Biomed. Eng. 2011, 58, 1846-1854.

Vallejos, S.; Munoz, A.; Ibeas, S.; Serna, F.; Garcia, F. C.; Garcia, J. M. Solid sensory polymer substrates for the quantification of iron in blood, wine and water by a scalable RGB technique. J. Mater. Chem. A. 2013, 1, 15435-15441.

Walczak, R.; Kruger, J.; Moynihan, S. A miniaturised image based fluorescence detection system for point-of-care-testing of cocaine abuse. Meas. Sci. Technol. 2015, 26, 15 .

Wang, S. Q.; Zhao, X. H.; Khimji, I.; Akbas, R.; Qiu, W. L.; Edwards, D.; Cramer, D. W.; Ye, B.; Demirci, U. Integration of cell phone imaging with microchip ELISA to detect ovarian cancer HE4 biomarker in urine at the point-of-care. Lab Chip 2011, 11, 3411-3418.

Wang, Y.; Li, Y. Y.; Bao, X.; Han, J.; Xia, J. C.; Tian, X. Y.; Ni, L. A smartphone-based colorimetric reader coupled with a remote server for rapid on-site catechols analysis. Talanta 2016a, 160, 194-204.

Wang, Y.; Liu, X. H.; Chen, P.; Tran, N. T.; Zhang, J. L.; Chia, W. S.; Boujday, S.; Liedberg, B. Smartphone spectrometer for colorimetric biosensing. Analyst 2016b, 141, 3233-3238.

Wang, L. J.; Chang, Y. C.; Sun, R. R.; Li, L. A multichannel smartphone optical biosensor for high-throughput point-of-care diagnostics. Biosens. Bioelectron. 2017, 87, 686-692.

Wei, Q.; Qi, H.; Luo, W.; Tseng, D.; Ki, S. J.; Wan, Z.; Cöröcs, Z.; Bentolila, L. A.; Wu, T.-T.; Sun, R.; Ozcan, A. Fluorescent imaging of single nanoparticles and viruses on a smart phone. Acs Nano. 2013, 7, 9147-9155.

Wei, Q.; Nagi, R.; Sadeghi, K.; Feng, S.; Yan, E.; Ki, S. J.; Caire, R.; Tseng, D.; Ozcan, A. Detection and spatial mapping of mercury contamination in water samples using a smart-phone. Acs Nano. 2014, 8, 1121-1129.

Windmiller, J. R.; Wang, J. Wearable electrochemical sensors and biosensors: a review. Electroanalysis. 2013, 25, $29-46$.

Xu, W.; Lu, S.; Chen, Y.; Zhao, T.; Jiang, Y.; Wang, Y.; Chen, X. Simultaneous color sensing of O2 and pH using a smartphone. Sensors Actuators $B$ Chem. 2015, 220, 326-330.

Yazawa, Y.; Oonishi, T.; Watanabe, K.; Shiratori, A.; Funaoka, S.; Fukushima, M. System-on-fluidics immunoassay device integrating wireless radio-frequency-identification sensor chips. J. Biosci. Bioeng. 2014, 118, 344-349.

Yu, H. J.; Tan, Y. F.; Cunningham, B. T. Smartphone Fluorescence Spectroscopy. Anal. Chem. 2014, 86, 8805-8813.

Zangheri, M.; Cevenini, L.; Anfossi, L.; Baggiani, C.; Simoni, P.; Di Nardo, F.; Roda, A. A simple and compact smartphone accessory for quantitative chemiluminescence-based lateral flow immunoassay for salivary cortisol detection. Biosens. Bioelectron. 2015, 64, 63-68.

Zhang, D.; Liu, Q. Biosensors and bioelectronics on smartphone for portable biochemical detection. Biosens. Bioelectron. 2016, 75, 273-284. 